Draft version August 8, 2018

Preprint typeset using $\mathrm{LT}_{\mathrm{E} X} \mathrm{X}$ style emulateapj v. 5/2/11

\title{
THE TIP OF THE RED GIANT BRANCH DISTANCES TO TYPE IA SUPERNOVA HOST GALAXIES. IV. COLOR DEPENDENCE AND ZERO-POINT CALIBRATION
}

\author{
In Sung Jang and Myung Gyoon Lee \\ Astronomy Program, Department of Physics and Astronomy, Seoul National University, Gwanak-gu, Seoul 151-742, Korea \\ Draft version August 8, 2018
}

\begin{abstract}
We present a revised TRGB calibration, accurate to $2.7 \%$ of distance. A modified TRGB magnitude corrected for the color dependence of the TRGB, the $Q T$ magnitude, is introduced for better measurement of the TRGB. We determine the color-magnitude relation of the TRGB from photometry of deep images of HST/ACS fields around eight nearby galaxies. The zero-point of the TRGB at the fiducial metallicity $\left([\mathrm{Fe} / \mathrm{H}]=-1.6\left((V-I)_{0, T R G B}=1.5\right)\right)$ is obtained from photometry of two distance anchors, NGC 4258 (M106) and the LMC, to which precise geometric distances are known: $M_{Q T, T R G B}=-4.023 \pm 0.073 \mathrm{mag}$ from NGC 4258 and $M_{Q T, T R G B}=-4.004 \pm 0.096 \mathrm{mag}$ from the LMC. A weighted mean of the two zero-points is $M_{Q T, T R G B}=-4.016 \pm 0.058$ mag. Quoted uncertainty is $\sim 2$ times smaller than those of the previous calibrations. We compare the empirical TRGB calibration derived in this study with theoretical stellar models, finding that there are significant discrepancies, especially for red color $\left((\mathrm{F} 606 \mathrm{~W}-\mathrm{F} 814 \mathrm{~W})_{0} \gtrsim 2.5\right)$. We provide the revised TRGB calibration in several magnitude systems for future studies.

Subject headings: galaxies: distances and redshifts — galaxies: stellar content — stars : Population II
\end{abstract}

\section{INTRODUCTION}

The Tip of the Red Giant Branch (TRGB) represents the brightest part of the Red Giant Branch (RGB) in the color-magnitude diagrams (CMDs) of old stellar systems such as globular clusters and halos in galaxies. It corresponds to the core-He flash point in the evolutionary stages of low mass stars. An implication of the TRGB as a distance indicator was suggested first by Baade (1944). In his pioneering paper introducing the concept of stellar populations I and II, he investigated the red-sensitive plates of three early type stellar systems, M32, NGC 205, and the central region (bulge) of M31, concluding that the brightest stars (RGB stars) in these three systems have similar magnitudes and colors. Sandage (1971) pointed out that photographs of the Local Group galaxies invariably show surrounding red stars, which have similar absolute magnitudes and colors. Based on the Cepheid distances to three Local Group galaxies (M31, M33 and IC 1613), he derived a mean absolute magnitude of these brightest red stars to be $M_{V}=-3.0 \pm 0.2$ mag

Mould et al. (1983, 1984) and Mould \& Kristian (1986) carried out CCD photometry of four Local Group galaxies (M31, M33, NGC 147 and NGC 205) and determined the TRGB distances to these galaxies, which are not much different from previous distance estimates to M31 and M33 based on Cepheid variables. They adopted a bolometric magnitude of the TRGB $\left(M_{\mathrm{bol}} \sim-3.5\right.$ mag) given by Frogel et al. (1983). Freedman (1988b) presented CCD photometry of two fields in IC 1613. Color-magnitude diagrams (CMDs) of these fields show a clear RGB population and the TRGB. Adopting the bolometric magnitude the TRGB $\left(M_{\mathrm{bol}} \sim-3.5 \mathrm{mag}\right)$, she derived remarkably similar TRGB distances between the two fields, showing only 0.04 mag difference. Moreover, the TRGB distance from her photometry is in ex-

isjang@astro.snu.ac.kr, mglee@astro.snu.ac.kr cellent agreement with the Cepheid distance to IC 1613 obtained by Freedman (1988a).

A feasibility of the TRGB as a robust distance indicator was established by Lee et al. (1993). They investigated empirical RGB loci of the Milky Way globular clusters (Da Costa \& Armandroff 1990) and Yale theoretical stellar isochrones (Green et al.|1987), finding that the $I$-band magnitude of the TRGB is almost constant with variation smaller than $0.1 \mathrm{mag}\left(M_{I, \mathrm{TRGB}}=\right.$ $-4.0 \pm 0.1)$ for low metallicity $([\mathrm{Fe} / \mathrm{H}] \leq-0.7)$ or for age older than 3 Gyr. Also, they applied a Sobel kernel for detecting the TRGB from the discontinuity of the $I$-band luminosity function. They found that a comparison of the TRGB distances to 12 Local Group galaxies with the distances based on Cepheid and RRLyrae variables shows a good agreement, showing that the precision of the TRGB is comparable to these primary distance indicators. With the TRGB, distances to more than three hundreds of nearby galaxies have been measured (Freedman \& Madore 2010; Dalcanton et al. 2009; Radburn-Smith et al. 2011; Tully et al. 2013; Lee \& Jang 2013, 2016).

In comparison with the Cepheid variables, which are a well known primary distance indicator, the TRGB has several advantages. First, the TRGB method uses Population II RGB stars. It enables to measure distances to both early type galaxies and late type galaxies, while Cepheid variables can be used only for late type galaxies. Second, the TRGB method mainly uses RGB stars in the halo regions, where stellar densities are low and internal extinctions are negligible, while Cepheid variables are located in the star-forming regions where internal extinctions are often significant. Third, the TRGB stars are non-variable stars, so that a single epoch observation is enough to get distances, while Cepheids require many epochs of observations. Fourth, the metallicity dependence of the $I$-band magnitude of the TRGB is known to be small for low metallicity Lee et al. 1993; 
TABLE 1

A Summary of HST Observations of the TRGB Calibration Sample

\begin{tabular}{|c|c|c|c|c|c|c|c|c|}
\hline \multirow[t]{2}{*}{ Target } & \multirow[t]{2}{*}{ Field } & \multirow{2}{*}{$\begin{array}{l}\text { R.A. } \\
\text { (J2000.0) }\end{array}$} & \multirow{2}{*}{$\begin{array}{l}\text { Decl. } \\
(\mathrm{J} 2000.0)\end{array}$} & \multirow[t]{2}{*}{ Instrument } & \multicolumn{3}{|c|}{ Exposure time } & \multirow{2}{*}{ Prop. ID } \\
\hline & & & & & F555W & F606W & F814W & \\
\hline \multirow[t]{2}{*}{ M105 } & $\mathrm{F} 1$ & 104801.53 & 123228.2 & $\mathrm{ACS} / \mathrm{WFC}$ & & $9,775 \mathrm{~s}$ & $9,775 \mathrm{~s}$ & 10413 \\
\hline & $\mathrm{F} 2$ & 104800.20 & 123206.8 & $\mathrm{ACS} / \mathrm{WFC}$ & & $9,775 \mathrm{~s}$ & $9,775 \mathrm{~s}$ & 10413 \\
\hline \multirow[t]{2}{*}{ NGC 3384} & F1 & 104826.03 & 123950.6 & $\mathrm{ACS} / \mathrm{WFC}$ & & $14,575 \mathrm{~s}$ & $14,575 \mathrm{~s}$ & 10413 \\
\hline & $\mathrm{F} 2$ & 104822.38 & 123908.6 & $\mathrm{ACS} / \mathrm{WFC}$ & & $9,825 \mathrm{~s}$ & $9,825 \mathrm{~s}$ & 10413 \\
\hline \multirow[t]{3}{*}{ M81 } & $\mathrm{F} 1$ & 095434.56 & $\begin{array}{lll}69 & 16 & 50.9\end{array}$ & $\mathrm{ACS} / \mathrm{WFC}$ & & $24,232 \mathrm{~s}$ & $29,953 \mathrm{~s}$ & 10915 \\
\hline & $\mathrm{F} 2$ & 095413.96 & $\begin{array}{lll}69 & 05 & 42.6\end{array}$ & $\mathrm{ACS} / \mathrm{WFC}$ & & $5,354 \mathrm{~s}$ & $5,501 \mathrm{~s}$ & 10136 \\
\hline & F3 & 095701.20 & 685555.6 & $\mathrm{ACS} / \mathrm{WFC}$ & & $1,580 \mathrm{~s}$ & $1,595 \mathrm{~s}$ & 10584 \\
\hline NGC 3377 & F1 & 104748.11 & $1355 \quad 45.2$ & $\mathrm{ACS} / \mathrm{WFC}$ & & $38,500 \mathrm{~s}$ & $22,260 \mathrm{~s}$ & 9811 \\
\hline NGC 253 & F1 & 004819.46 & $\begin{array}{lll}-25 & 08 & 47.4\end{array}$ & $\mathrm{ACS} / \mathrm{WFC}$ & & $2,283 \mathrm{~s}$ & $2,253 \mathrm{~s}$ & 10915 \\
\hline NGC 4258 & $\mathrm{~F} 1$ & 121918.37 & 472013.8 & $\mathrm{ACS} / \mathrm{WFC}$ & $5,700 \mathrm{~s}$ & & $2,600 \mathrm{~s}$ & 9477 \\
\hline \multirow[t]{3}{*}{ NGC 300} & F1 & 005447.98 & -374051.6 & $\mathrm{ACS} / \mathrm{WFC}$ & & $1,515 \mathrm{~s}$ & $1,542 \mathrm{~s}$ & 10915 \\
\hline & $\mathrm{F} 2$ & 005434.70 & -373925.4 & $\mathrm{ACS} / \mathrm{WFC}$ & & $1,515 \mathrm{~s}$ & $1,542 \mathrm{~s}$ & 10915 \\
\hline & F3 & 005421.37 & -373756.3 & $\mathrm{ACS} / \mathrm{WFC}$ & & $1,515 \mathrm{~s}$ & $1,542 \mathrm{~s}$ & 10915 \\
\hline NGC 3077 & $\mathrm{~F} 1$ & 100328.41 & 684352.8 & $\mathrm{ACS} / \mathrm{WFC}$ & & $1,596 \mathrm{~s}$ & $1,622 \mathrm{~s}$ & 10915 \\
\hline
\end{tabular}

Rizzi et al. 2007; Madore et al. 2009; Bellazzini 2008), while the metallicity effect on the optical luminosity of Cepheids is controversial (Gerke et al. 2011).

On the other hand, the TRGB has a disadvantage that its luminosity is relatively fainter than those of the bright Cepheid variables. The absolute magnitude of the brightest Cepheid variable is estimated to be $M_{V} \sim-7$ mag, about $4.5 \mathrm{mag}$ brighter than that of the TRGB, $M_{V} \sim-2.5 \mathrm{mag}$. This difference becomes smaller in the longer wavelength: $\Delta$ mag $=3.9,3.1,2.6$ and 2.6 mag in $I, J, H$, and $K_{s}$ bands, respectively (Macri et al. 2006, 2015, assuming the maximum period of Cepheid variables $\left(P_{\max }=100\right.$ days $\left.)\right)$.

Taking advantage of the TRGB, we started a project, the TRGB distances to SN host galaxies in the Universe (TIPSNU), to improve luminosity calibration of SNe. Lee \& Jang (2012) (Paper I) presented a TRGB distance to nearby spiral galaxy M101 hosting SN $2011 \mathrm{fe}$. Lee \& Jang (2013) (Paper II) determined TRGB distances to M66 and M96 in the Leo I group hosting two SNe Ia, SN 1989B and SN 1998bu. Later, Jang \& Lee (2015) (Paper III) obtained TRGB distances to two galaxies, NGC 4038/39 hosting SN 2007sr and NGC 5584 hosting SN 2007af. Combining the TRGB distances and optical light curves of the five SNe Ia, Jang \& Lee (2015) derived a value of the Hubble constant, $H_{0}=$ $69.8 \pm 2.6 \pm 3.9 \mathrm{~km} \mathrm{~s}^{-1} \mathrm{Mpc}^{-1}$. This value is similar to those from the cosmic microwave background (Bennett et al. 2014; Planck Collaboration et al. 2015) but still agrees with in errors from those of Cepheid calibrated SNe Ia (Riess et al. 2011; Freedman et al. 2012; Riess et al. 2016). In order to make better estimation of $H_{0}$, it is needed to reduce both random and systematic uncertainties.

This paper, the fourth paper of our series, is aiming to refine two issues in the TRGB method: a calibration of the color (metallicity) - luminosity relation and a calibration of the zero-point of the TRGB magnitude. Both calibrations are important to reduce the systematic uncertainty of the TRGB distance estimates and of the value of the Hubble constant. Previous color dependence calibrations were based on theoretical stellar models (Salaris \& Cassisi 1997; Fusco et al. 2012), or on a small number of stellar systems (Milky Way globu- lar clusters or nearby galaxies) showing a narrow RGB color range (Lee et al.|1993; Bellazzini et al.|2001, 2004; Rizzi et al. 2007; Bellazzini 2008; Madore et al. 2009). These calibrations show a significant discrepancy of the color - luminosity relation ranging from flat to steep (as shown in the Discussion section).

Zero-point calibrations of the TRGB in previous studies were done with theoretical models (Salaris \& Cassisi 1997; Fusco et al. 2012) or observational approaches (Lee et al. 1993; Ferrarese et al.|2000; Bellazzini et al. 2001, 2004; Rizzi et al.|2007; Bellazzini 2008; Tammann et al. 2008; Madore et al. 2009). Various distance indicators (RR Lyrae stars, Cepheids, eclipsing binary stars, and horizontal branch stars) have been used for this calibration. However, derived values show a non-negligible scatter with a large uncertainty, as shown in the Discussion section.

This paper is composed of as follows. In Section 2 we describe how we derive color (metallicity) - luminosity relation of the TRGB. Target galaxy selection criteria and data reduction methods will be presented. $\S 3$ shows the zero-point calibration of the TRGB based on two distance anchors: NGC 4258 and the LMC. A summary of the revised TRGB calibration including various filter systems is shown in $\S 4$. We discuss implications of our results in $\S 5$ and summarize primary results in the final section.

\section{COLOR DEPENDENCE CALIBRATION}

\subsection{Target Selection and Data Reduction}

Optimal target galaxies and target fields for the color (metallicity) dependence calibration of the TRGB are expected to satisfy the following criteria: 1) showing a wide color range of the TRGB, covering from the metal poor (blue) RGB stars to the metal rich (red) RGB stars, 2) allowing a high signal to noise detection of RGB stars, 3) showing a dominant old RGB population, and 4) being observed with the same instrument to reduce any chance for increasing instrument-dependent uncertainties (e.g. photometric transformation uncertainties). Considering these selection criteria, we selected 14 fields around 8 nearby galaxies for which HST/Advanced Camera for Surveys $(A C S)$ images are available in the archive: M105 (E1), NGC 3384 (SB0), M81 (SA(s)AB), NGC 3377 (E5- 

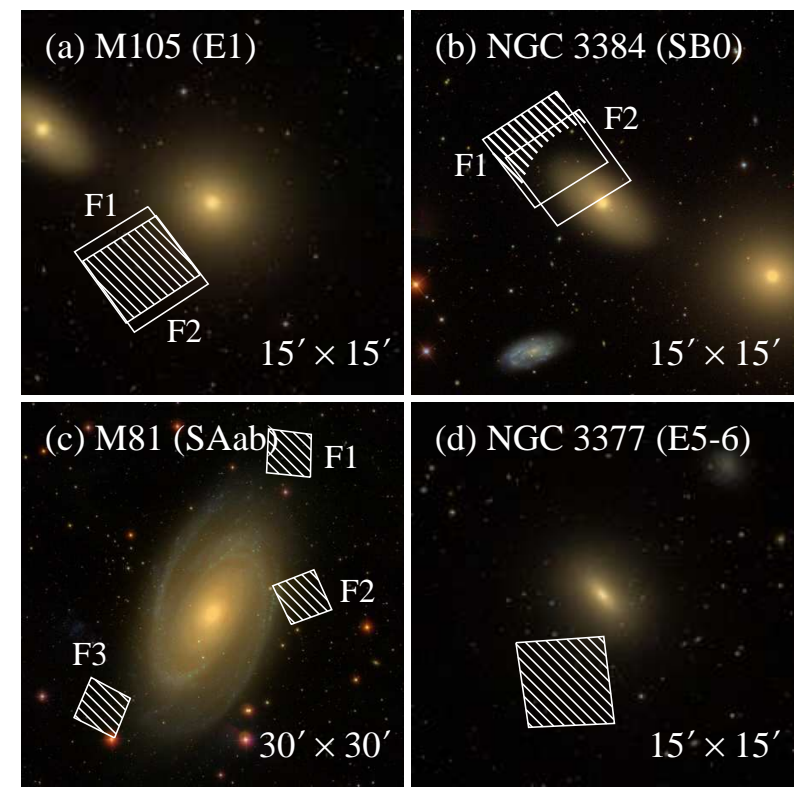

(d) NGC 3377 (E5-6)
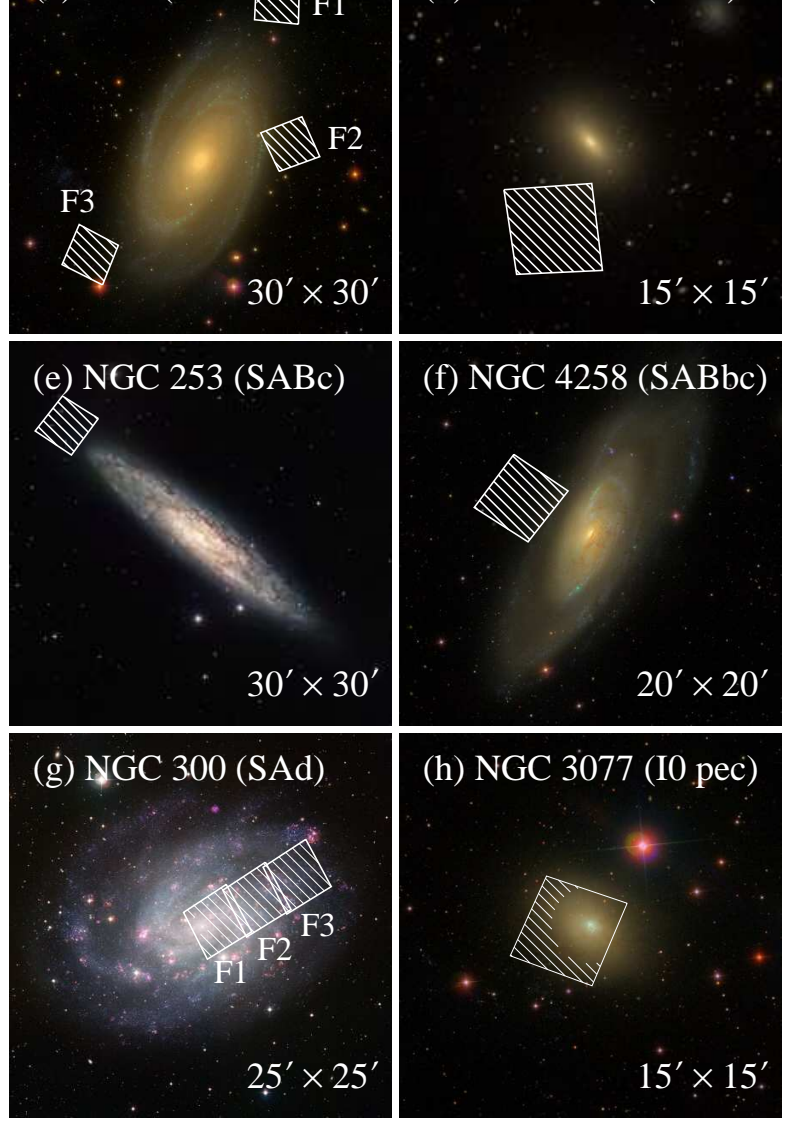

FIG. 1.- Finding charts for eight galaxies used for the color dependence calibration of the TRGB: M105 (a), NGC 3384 (b) M81 (c), NGC 3377 (d), NGC 253 (e), NGC 4258 (f), NGC 300 (g), and NGC 3077 (h). HST/ACS fields used in this study are marked on the color maps of the Sloan Digital Sky Survey (SDSS) or public images provided by the European Southern Observatory (ESO). The regions we used for the TRGB calibration are indicated by the hatched regions.

6), NGC $253(\mathrm{SAB}(\mathrm{s}) \mathrm{c}), \mathrm{NGC} 4258$ (SAB(s)bc), NGC 300 (SA(s)d), and NGC 3077 (I0 pec).

These galaxies have various morphological types from elliptical galaxies to spiral and irregular galaxies. Identifications of the HST fields in each galaxy are shown in Figure 11 These HST fields are located from the center to outer regions in the target galaxies. Note that the three fields in NGC 300 are located in the disk. Rizzi et al. (2007) used two HST/ACS fields in other positions of the NGC 300 disk for their TRGB color calibration. For this reason, we also included disk fields of NGC 300 for which deeper HST/ACS images are available. Table 1 lists a summary of $H S T / A C S$ observa- tions of the target galaxies. Exposure times are ranging from $1,515 \mathrm{~s}$ to $38,500 \mathrm{~s}$, long enough to detect resolved red giants. It is noted that NGC 4258 was observed with F555W filter, while other galaxies were observed with F606W filter.

We obtained ACS images of the fields from the $H S T$ archive and constructed deep master drizzled images, following the procedures described in Jang \& Lee (2015). In our previous studies (Lee \& Jang 2012, 2013; Jang \& Lee 2015), we carried out point spread function (PSF) photometry using DAOPHOT in IRAF package (Stetson 1987). We noted, however, that most of the previous and on-going studies for the calibration of SNe Ia (e.g. the Hubble Key Project (Freedman et al. 2001), the SN HST project (Sandage et al. 2006), the SH0ES Project (Riess et al. 2011), and the CarnegieChicago Hubble Program (Beaton et al. 2016)) have been done with the standalone version of DAOPHOT (Stetson 1987). Moreover, the Optical Gravitational Lensing Experiment (OGLE) photometric data of the LMC (Ulaczyk et al. 2012), which we used in the zeropoint calibration of the TRGB (see Section 3.2) is also based on the standalone version of DAOPHOT. Thus, we use the standalone version of DAOPHOT in this study, to reduce software dependent uncertainty as much as possible. Our previous results based on IRAF/DAOPHOT will be updated in the upcoming paper (Jang \& Lee 2016, in preparation). A single pass sequence (DAOFIND, PHOT, ALLSTAR and ALLFRAME) of PSF photometry was carried out. We used PSF images produced from isolated bright stars in each $A C S$ field. We adopted updated photometric zero-points and aperture correction values provided by the STScI webpag®.

\subsection{CMDs of Resolved Stars and the TRGB detection}

In Figure 2 (a, c, e, g, i, k, m, and o), we display CMDs of the resolved stars in the selected regions, marked by hatched regions in Figure 1, of each galaxy. The central regions of NGC $3384\left(R \leq 3^{\prime}\right)$, NGC 300 $\left(R \leq 0^{\prime} \cdot 6\right)$, and NGC $3077\left(R \leq 1^{\prime} \cdot 5\right)$ were discarded in the analysis because of serious crowding due to high stellar densities. The most prominent feature in all CMDs is a distinguishable RGB. The width of the RGBs shows a large variation: from narrow RGBs for low mass galaxies (NGC 300 and NGC 3077) to broad RGBs for high mass galaxies (M105 and M81). All CMDs show also a small population of asymptotic giant branch (AGB) stars. The CMD of the disk fields in NGC 300 shows not only a strong old RGB but also the presence of red core helium burning stars with much younger age at (F606W$\mathrm{F} 814 \mathrm{~W})_{0} \approx 1.0$.

We detected the TRGB by applying the Sobel edge detection algorithm with a kernel of $[-1,-2,-1,0,+1$, $+2,+1]$ and a bin size of 0.05 mag. In order to check the color-magnitude relation of the TRGB, we divided the stars into sub-groups according to their color with a color interval of $0.3 \mathrm{mag}$. In the case of NGC 300, we used a color interval of 0.15 mag because the CMD of this galaxy contains a much larger number of blue RGB stars. Figure 2 (b, d, f, h, j, l, n, and p) shows the TRGB detection results for the target galaxies. Edge detection responses are indicated by grey scale

\footnotetext{
${ }^{1}$ http://www.stsci.edu/hst/acs/analysis/zeropoints
} 

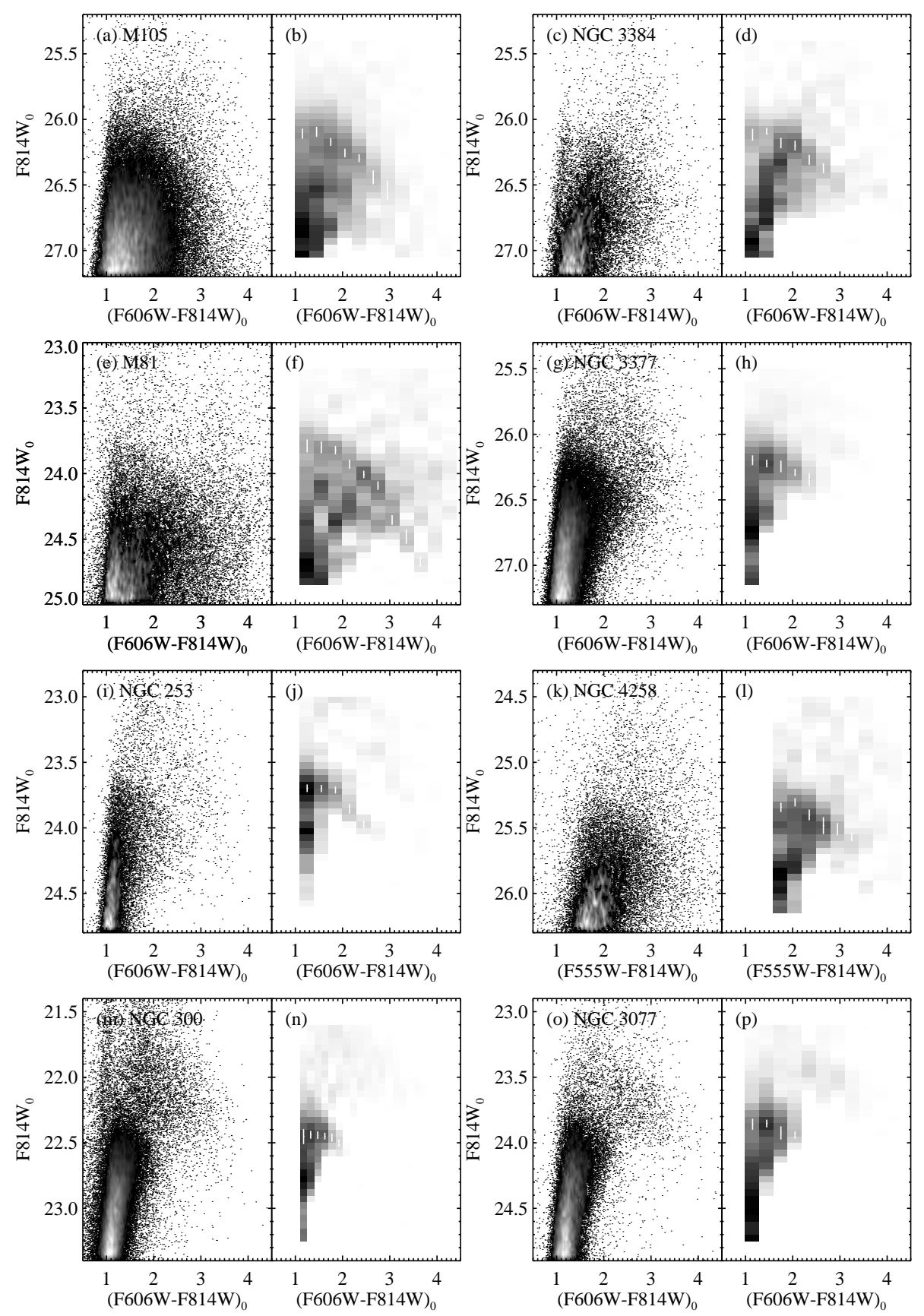

FIG. 2.- (Left windows in each panel) Foreground reddening-corrected CMDs of the resolved stars in eight galaxies used for the colordependence calibration. High density regions in each CMD are displayed as a density map (Hess diagram). (Right windows in each panel) Maps showing the strength of edge detection responses. We divided the stars in each CMD into subgroups based on their color and applied an edge detection algorithm. Dark and pale regions in each map represent strong and weak edge detection responses, respectively. Estimated TRGB magnitudes and their errors for the subgroups are marked by white vertical lines. Note that (F555W - F814W) 0 colors were used for NGC 4258, while (F606W - F814W) o colors were used for the other galaxies.

maps: black for strong response (sharp discontinuity in the luminosity function) and white for weak response. The TRGB magnitudes and corresponding measurement uncertainties are determined through ten thousand simulations of bootstrap resampling. Derived values and errors are listed in Table 2 and also indicated by white vertical lines in each grey scale map. All eight galaxies show clearly color - magnitude relations of the TRGB: the TRGBs become fainter, as color increases.

Madore et al. (2009) pointed out that at least $400 \sim$
500 stars in the one mag interval below the TRGB are needed to detect the TRGB within the Poisson noise of $0.1 \mathrm{mag}$. Considering this, we set $100 \mathrm{RGB}$ stars in 0.2 mag interval below the TRGB as a criterion for analysis. We chose a narrower range of magnitude, to use photometry with higher completeness. Then we counted the number of RGB stars in each subgroup in each galaxy to check whether they have a sufficient number of RGB stars for precise determination of the TRGB or not. Only the cases satisfying our selection criteria above, as indi- 
TABLE 2

A Summary of the TrGB Magnitudes for the Color Dependence Calibration

\begin{tabular}{|c|c|c|c|c|c|}
\hline Galaxy & Color range & $\mathrm{F} 814 \mathrm{~W}_{0, T R G B}$ & Galaxy & Color range & TRGB \\
\hline \multirow{8}{*}{ M105 } & $(\mathrm{F} 606 \mathrm{~W}-\mathrm{F} 814 \mathrm{~W})_{0}$ & $F 814 W_{0}$ & \multirow{8}{*}{ NGC 253} & $(\mathrm{~F} 606 \mathrm{~W}-\mathrm{F} 814 \mathrm{~W})_{0}$ & $F 814 W_{0}$ \\
\hline & $1.0 \sim 1.3$ & $26.109 \pm 0.033$ & & $1.1 \sim 1.4$ & $23.698 \pm 0.021$ \\
\hline & $1.3 \sim 1.6$ & $26.095 \pm 0.033$ & & $1.4 \sim 1.7$ & $23.700 \pm 0.019$ \\
\hline & $1.6 \sim 1.9$ & $26.172 \pm 0.022$ & & $1.7 \sim 2.0$ & $23.711 \pm 0.017$ \\
\hline & $1.9 \sim 2.2$ & $26.256 \pm 0.026$ & & $2.0 \sim 2.3$ & $23.854 \pm 0.032$ \\
\hline & $2.2 \sim 2.5$ & $26.296 \pm 0.023$ & & $2.3 \sim 2.6$ & $23.974 \pm 0.020$ \\
\hline & $2.5 \sim 2.8$ & $26.442 \pm 0.048$ & & & \\
\hline & $2.8 \sim 3.1$ & $26.540 \pm 0.069$ & & & \\
\hline \multirow{7}{*}{ NGC 3384} & $(\mathrm{~F} 606 \mathrm{~W}-\mathrm{F} 814 \mathrm{~W})_{0}$ & $F 814 W_{0}$ & \multirow{7}{*}{ NGC 4258} & $(\mathrm{~F} 555 \mathrm{~W}-\mathrm{F} 814 \mathrm{~W})_{0}$ & $F 814 W_{0}$ \\
\hline & $1.0 \sim 1.3$ & $26.117 \pm 0.041$ & & $1.6 \sim 1.9$ & $25.343 \pm 0.029$ \\
\hline & $1.3 \sim 1.6$ & $26.091 \pm 0.017$ & & $1.9 \sim 2.2$ & $25.305 \pm 0.024$ \\
\hline & $1.6 \sim 1.9$ & $26.180 \pm 0.035$ & & $2.2 \sim 2.5$ & $25.401 \pm 0.036$ \\
\hline & $1.9 \sim 2.2$ & $26.200 \pm 0.032$ & & $2.5 \sim 2.8$ & $25.483 \pm 0.061$ \\
\hline & $2.2 \sim 2.5$ & $26.308 \pm 0.032$ & & $2.8 \sim 3.1$ & $25.510 \pm 0.042$ \\
\hline & $2.5 \sim 2.8$ & $26.372 \pm 0.033$ & & $3.1 \sim 3.4$ & $25.569 \pm 0.025$ \\
\hline \multirow{10}{*}{ M81 } & $(\mathrm{F} 606 \mathrm{~W}-\mathrm{F} 814 \mathrm{~W})_{0}$ & $F 814 W_{0}$ & \multirow{10}{*}{ NGC 300} & $(\mathrm{~F} 606 \mathrm{~W}-\mathrm{F} 814 \mathrm{~W})_{0}$ & $F 814 W_{0}$ \\
\hline & $1.1 \sim 1.4$ & $23.789 \pm 0.045$ & & $1.10 \sim 1.25$ & $22.453 \pm 0.053$ \\
\hline & $1.4 \sim 1.7$ & $23.799 \pm 0.041$ & & $1.25 \sim 1.40$ & $22.439 \pm 0.022$ \\
\hline & $1.7 \sim 2.0$ & $23.821 \pm 0.024$ & & $1.40 \sim 1.55$ & $22.442 \pm 0.020$ \\
\hline & $2.0 \sim 2.3$ & $23.928 \pm 0.027$ & & $1.55 \sim 1.70$ & $22.449 \pm 0.016$ \\
\hline & $2.3 \sim 2.6$ & $24.006 \pm 0.020$ & & $1.70 \sim 1.85$ & $22.461 \pm 0.022$ \\
\hline & $2.6 \sim 2.9$ & $24.092 \pm 0.026$ & & $1.85 \sim 2.00$ & $22.502 \pm 0.018$ \\
\hline & $2.9 \sim 3.2$ & $24.354 \pm 0.031$ & & & \\
\hline & $3.2 \sim 3.5$ & $24.480 \pm 0.051$ & & & \\
\hline & $3.5 \sim 3.8$ & $24.672 \pm 0.034$ & & & \\
\hline \multirow{6}{*}{ NGC 3377} & $(\mathrm{~F} 606 \mathrm{~W}-\mathrm{F} 814 \mathrm{~W})_{0}$ & $F 814 W_{0}$ & \multirow{6}{*}{ NGC 3077} & $(\mathrm{~F} 606 \mathrm{~W}-\mathrm{F} 814 \mathrm{~W})_{0}$ & $F 814 W_{0}$ \\
\hline & $1.0 \sim 1.3$ & $26.198 \pm 0.032$ & & $1.0 \sim 1.3$ & $23.859 \pm 0.037$ \\
\hline & $1.3 \sim 1.6$ & $26.223 \pm 0.021$ & & $1.3 \sim 1.6$ & $23.853 \pm 0.021$ \\
\hline & $1.6 \sim 1.9$ & $26.245 \pm 0.040$ & & $1.6 \sim 1.9$ & $23.925 \pm 0.046$ \\
\hline & $1.9 \sim 2.2$ & $26.291 \pm 0.019$ & & $1.9 \sim 2.1$ & $23.941 \pm 0.020$ \\
\hline & $2.2 \sim 2.5$ & $26.345 \pm 0.045$ & & & \\
\hline
\end{tabular}

cated by white vertical lines in Figure 2 were used in the following analysis.

\subsection{Color Transformation between $\mathrm{F} 555 \mathrm{~W}-\mathrm{F} 814 \mathrm{~W}$ and $\mathrm{F} 606 \mathrm{~W}-\mathrm{F} 814 \mathrm{~W}$}

NGC 4258 is an important galaxy for calibrations of both the color dependence and the zero-point, because it shows a broad range of RGB color and hosts a megamaser, which is useful for geometric distance estimation. NGC 4258 was observed with F555W and F814W filters. However, the other galaxies used for color dependence calibration were observed with F606W and F814W filters. Therefore, the color transformation between $\mathrm{F} 555 \mathrm{~W}-\mathrm{F} 814 \mathrm{~W}$ and $\mathrm{F} 606 \mathrm{~W}-\mathrm{F} 814 \mathrm{~W}$ is needed for a direct comparison. Sirianni et al. (2005) provides photometric transformations from ACS to Johnson-Cousins systems. With these relations, F555W and F814W magnitudes can be converted to $V$ and $I$ magnitudes, respectively. Similarly, $V$ and $I$ magnitudes can also be converted to $\mathrm{F} 555 \mathrm{~W}$ and $\mathrm{F} 814 \mathrm{~W}$ magnitudes, respectively. This method enables the color transformation between the two ACS colors. However, applying transformation twice would increase a systematic uncertainty.

In Paper III (Jang \& Lee 2015), we presented F555W, F606W, and F814W bands photometry of two Milky Way globular clusters, NGC 2419 and 47 Tuc. Using the photometric catalogs of the stars in the two globular clusters obtained from our previous study, we derive a direct color transformation. We selected resolved stars in each globular cluster with the following criteria: $R \geq 1^{\prime}$ and $18.5<\mathrm{F} 814 \mathrm{~W} \leq 21.0 \mathrm{mag}$ for NGC 2419 , and $R \geq 7^{\prime}$ and $19.5<\mathrm{F} 814 \mathrm{~W} \leq 22.0 \mathrm{mag}$ for 47

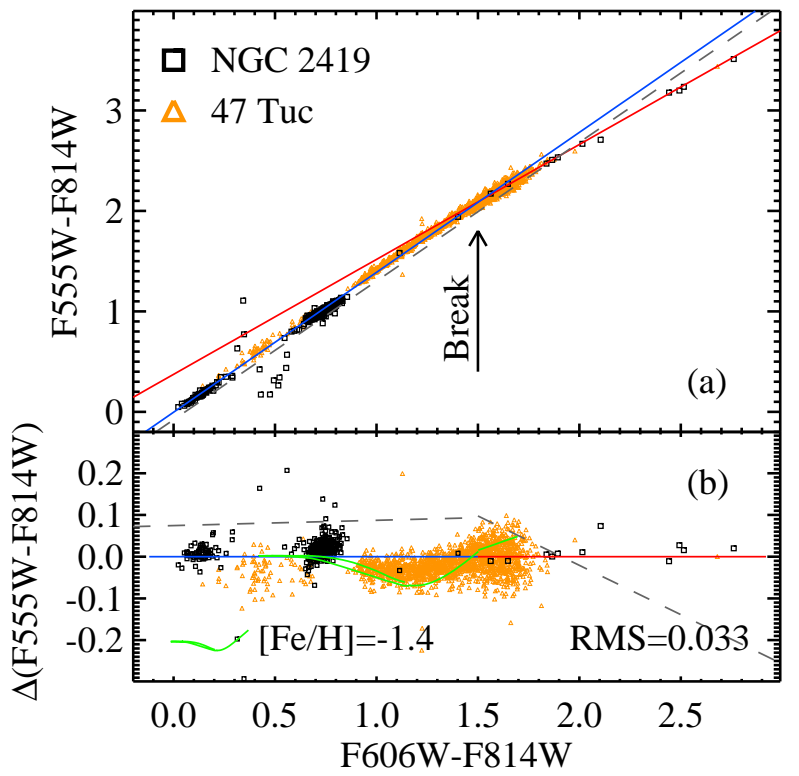

Fig. 3.- (a) Color difference between F606W - F814W and $\mathrm{F} 555 \mathrm{~W}-\mathrm{F} 814 \mathrm{~W}$ as a function of $\mathrm{F} 606 \mathrm{~W}-\mathrm{F} 814 \mathrm{~W}$ color for the stars in NGC 2419 (open squares) and 47 Tuc (open triangles). Blue and red solid lines show double linear fits to the observed colors: the blue stars $(\mathrm{F} 606 \mathrm{~W}-\mathrm{F} 814 \mathrm{~W} \leq 1.5$, blue line $)$ and the red stars $(\mathrm{F} 606 \mathrm{~W}-\mathrm{F} 814 \mathrm{~W}>1.5$, red line). (b) Residual F555WF814W color after subtraction of the linear fits for the blue and the red stars. The curved line indicates 12 Gyr stellar isochrone for a metallicity of $[\mathrm{Fe} / \mathrm{H}]=-1.4$, which is a mean metallicity of the two globular clusters, provided by the Dartmouth group. The gray dashed line represents the color relation expected from the photometric transformations given in Sirianni et al. (2005), which shows a strong difference with respect to the observed stars. 


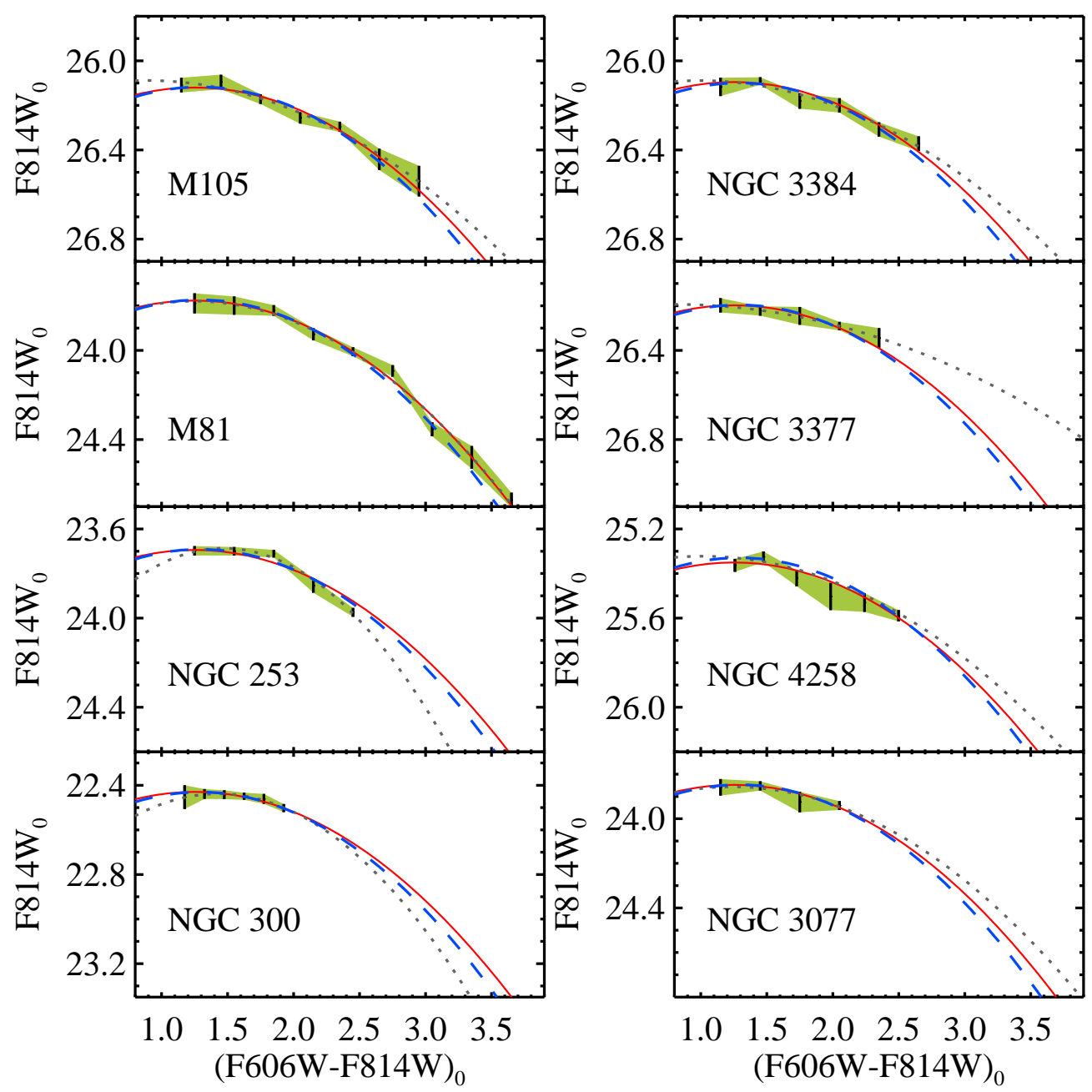

FIG. 4.- F814W $-(\mathrm{F} 606 \mathrm{~W}-\mathrm{F} 814 \mathrm{~W})_{0}$ color-magnitude relations of the TRGB for eight galaxies. Green shaded regions with vertical short lines represent the measured TRGB magnitudes with errors. Three best fit results with a quadratic equation are overlayed: constraining the same curvature parameters $(\alpha$ and $\beta$ ) for entire color range of all eight galaxies (red solid lines), the same fit results for the red solid lines except for the use of the blue color range $(\mathrm{F} 606 \mathrm{~W}-\mathrm{F} 814 \mathrm{~W} \leq 2.5)$ only (blue dashed lines), and free curvature parameters for entire color range of each galaxy (grey dotted lines).

Tuc (see Figure 4 of Jang \& Lee (2015)). A comparison of two ACS colors from the selected stars is shown in Figure 3(a). The F606W - F814W color ranges from 0.0 to $2.8 \mathrm{mag}$. A large scatter of NGC 2419 stars at F606W - F814W $\sim 0.5 \mathrm{mag}$ is probably due to the $\mathrm{RR}$ Lyrae variables. The distribution of stars can be fitted well by two linear relations (blue and red solid lines) with a break at $\mathrm{F} 606 \mathrm{~W}-\mathrm{F} 814 \mathrm{~W}=1.5 \mathrm{mag}$ : mouth models (Dotter et al. 2008) as a curved line. We also overlay a relation from the observational transformations given in Sirianni et al. (2005) (dot-dashed line). Theoretical model agrees very well with the observed stars. However, the relation from Sirianni et al. (2005) shows a systematic offset of $\sim 0.1 \mathrm{mag}$. We use double linear relations derived in this study in the following analysis.

$(\mathrm{F} 555 \mathrm{~W}-\mathrm{F} 814 \mathrm{~W})=(1.393 \pm 0.003)(\mathrm{F} 606 \mathrm{~W}-\mathrm{F} 814 \mathrm{~W})-(0.004 \pm 0.004)_{2.4}$

for $\mathrm{F} 606 \mathrm{~W}-\mathrm{F} 814 \mathrm{~W} \leq 1.5 \mathrm{mag}$, and

$(\mathrm{F} 555 \mathrm{~W}-\mathrm{F} 814 \mathrm{~W})=(1.148 \pm 0.021)(\mathrm{F} 606 \mathrm{~W}-\mathrm{F} 814 \mathrm{~W})+($

for $\mathrm{F} 606 \mathrm{~W}-\mathrm{F} 814 \mathrm{~W}>1.5 \mathrm{mag}$.

Figure 3(b) displays F555W - F814W color difference between the observed stars and the linear relation of the blue $(\mathrm{F} 606 \mathrm{~W}-\mathrm{F} 814 \mathrm{~W} \leq 1.5 \mathrm{mag})$ and the red $(\mathrm{F} 606 \mathrm{~W}-\mathrm{F} 814 \mathrm{~W}>1.5 \mathrm{mag})$ stars. The root mean square of stars over the entire color range $(0.0<$ F606W-F814W $<2.8$ ) is $0.033 \mathrm{mag}$. We overlay a stellar isochrone for 12 Gyr age, and $[\mathrm{Fe} / \mathrm{H}]=-1.4$, which is a mean metallicity of the two globular clusters, in the Dart-
In Figure 4. we plotted the $(\mathrm{F} 606 \mathrm{~W}-\mathrm{F} 814 \mathrm{~W})_{0}$ color and $\mathrm{F} 814 \mathrm{~W}_{0}$ magnitude relations of the TRGB stars in (0.336 erght 35 alaxies. The $(\mathrm{F} 555 \mathrm{~W}-\mathrm{F} 814 \mathrm{~W})_{0}$ color of NGC 4258 was converted to $(\mathrm{F} 606 \mathrm{~W}-\mathrm{F} 814 \mathrm{~W})_{0}$ color using the transformation described in Section 2.3. The TRGB magnitudes show a clear non-linear relation as a function of color. We use an equation below, keeping quadratic terms for the approximation of the TRGB:

$\mathrm{F} 14 \mathrm{~W}_{\mathrm{TRGB}}=\alpha\left(\text { Color }_{\mathrm{TRGB}}-\gamma\right)^{2}+\beta\left(\right.$ Color $\left._{\mathrm{TRGB}}-\gamma\right)+\delta$

where $\gamma$ means a fiducial color at $[\mathrm{Fe} / \mathrm{H}]=-1.6$. In the case of $\mathrm{F} 606 \mathrm{~W}-\mathrm{F} 814 \mathrm{~W}$ combination, the value 
TABLE 3

Approximation of the Color - Magnitude Relation of the TrGB

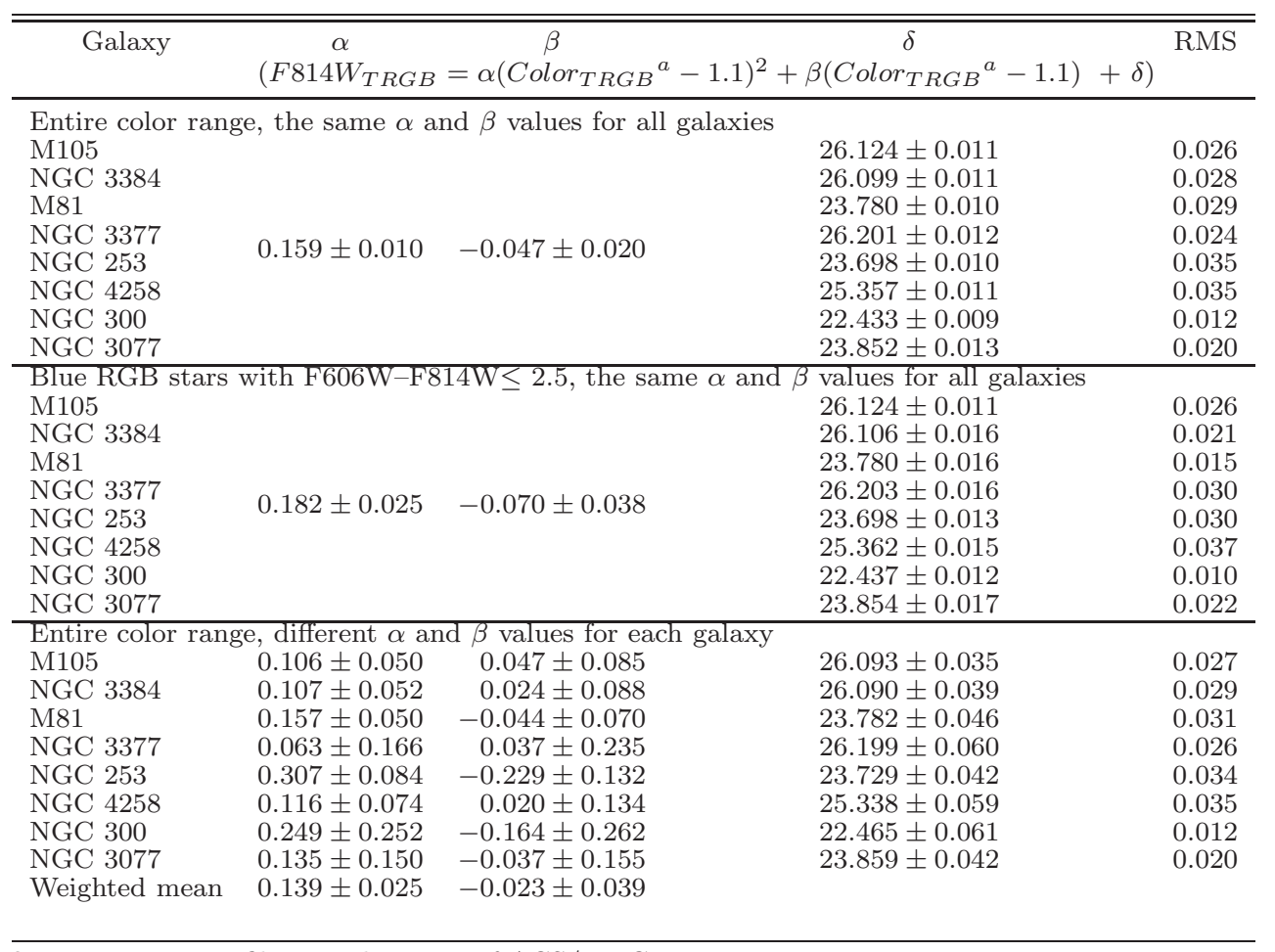

${ }^{\mathrm{a}} \mathrm{F} 606 \mathrm{~W}-\mathrm{F} 814 \mathrm{~W}$ filter combination of ACS/WFC

of $\gamma$ corresponds to 1.1. Coefficients $\alpha$ and $\beta$ determine the shape of the relation, and $\delta$ reflects apparent magnitudes of the TRGB in each galaxy. Thus, values of $\alpha$ and $\beta$ are the same in all eight galaxies, but that of $\delta$ depends on galaxies. We carried out a multiparameter fitting for entire color range of the TRGB bins using the IDL/mpfitexpr to find the values of three coefficients and listed results in Table 3 . Derived values are $\alpha=0.159 \pm 0.010, \beta=-0.047 \pm 0.020$, and $\delta=22.4 \sim 26.2$, as plotted by solid lines in Figure 4

We investigated the variation of $\alpha$ and $\beta$ values depending on the selection of the TRGB color and the galaxies. When we use the TRGB color bins bluer than $(F 606 W-F 814 W)_{0}=2.5$ in the fit, we get $\alpha=0.182 \pm 0.025$ and $\beta=-0.070 \pm 0.038$ (shown as dashed lines in Figure 4), which agree well with those from the entire color bins. If we try fits for individual galaxies with the entire color bins, we obtain $\alpha=0.063$ to $0.307, \beta=-0.229$ to 0.047 (as shown by dotted lines in the figure). Weighted mean of the eight $\alpha$ and $\beta$ values are $\alpha=0.139 \pm 0.025$ and $\beta=-0.023 \pm 0.039$, which are similar to the results from the same curvature parameters for all eight galaxies. All the $\alpha$ and $\beta$ values from individual galaxies agree well within $1 \sigma$ level, with the weighted mean values, except for the values from NGC 253. NGC 253 gives $\alpha=0.307 \pm 0.084$ and $\beta=-0.229 \pm 0.132$, which show $2.0 \sigma$ and $1.6 \sigma$ differences, marginally agreeing with the weighted mean values. In the following analysis, we use $\alpha(=0.159 \pm 0.010)$ and $\beta(=-0.047 \pm 0.020)$ values from the fit of the entire color range of the TRGB bins with the same $\alpha$ and $\beta$ values of all eight galaxies.

We shifted the TRGB magnitudes and corresponding

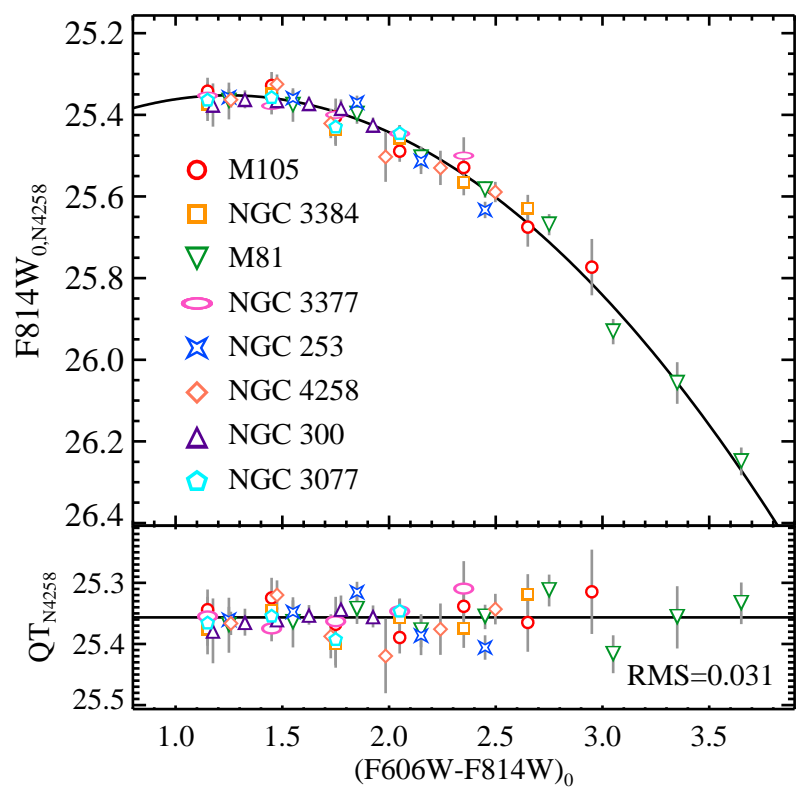

FIG. 5.- (Top) Combined F814W $\mathrm{W}_{0}$ magnitudes of the TRGB for eight galaxies as a function of $(\mathrm{F} 606 \mathrm{~W}-\mathrm{F} 814 \mathrm{~W})_{0}$ color. $\mathrm{F} 814 \mathrm{~W}_{0}$ magnitudes of each galaxy were shifted along the vertical direction, to be consistent with the result from NGC 4258. The solid line denotes the best fit result. (Bottom) Residuals between the best fit result and the data. Note that the RMS value for fitting is as small as 0.027 mag.

fit results of each galaxy along the vertical direction, to be consistent with the result from NGC 4258, and plotted them in Figure 5(a). All eight galaxies show reasonable agreements. The difference between the data points and 
the best fit result is shown in Figure 5(b). The root mean square of data is estimated to be 0.031 mag. It is similar to the mean measurement uncertainty of the TRGB.

We computed the correction parameters $\alpha, \beta$, and $\gamma$ for several different filter systems as well. The values for F555W - F814W combination in ACS/WFC were determined using the color transformation between F555W F814W and F606W - F814W shown in Section 2.3. The transformation was applied to seven galaxies that were observed with the F606W and F814W filters, excluding NGC 4258. Then we carried out multi-parameter fitting as done for F606W - F814W combination and obtained the values. In the fitting, we fixed $\gamma=1.6$, which corresponds to $[\mathrm{Fe} / \mathrm{H}]=-1.6$. Conversion relations described in Sirianni et al. (2005) were used to obtain the parameters in three photometric systems: F555W - F814W and $\mathrm{F} 606 \mathrm{~W}-\mathrm{F} 814 \mathrm{~W}$ combinations in WFPC2, and $V-I$ combination in the Johnson-Cousins system. Parameters for F555W - F814W and F606W - F814W combinations in WFC3/UVIS were derived by applying the photometric transformation between ACS/WFC to WFC3/UVIS described in Jang \& Lee (2015). Transformation uncertainty, $0.01 \sim 0.02$ mag were added in each calculation. Derived values are summarized in Table 6.

The color magnitude relation of the TRGB given in Figure 5 shows two interesting properties. First, at the blue color range of $F 606 W-F 814 W \leq 1.5$ (corresponding to $F 555 W-F 814 W \leq 2.1$ and $V-I \leq 1.9$ ), color magnitude relation of the TRGB is nearly flat in $\mathrm{F} 814 \mathrm{~W}_{0}-(\mathrm{F} 606 \mathrm{~W}-\mathrm{F} 814 \mathrm{~W})_{0} \mathrm{CMD}$, which means that additional color (metallicity) correction is not required in the TRGB measurement. If we use these blue RGB stars only in the TRGB measurement, distance modulus can be derived directly by adding the zero-point of the TRGB in F814W:

$$
(m-M)_{0}=F 814 W_{0, T R G B}+M_{F 814 W, T R G B} .
$$

Hereafter, we call this the blue $I$ calibration.

Second, the red color range with $F 606 W-F 814 W>$ 1.5 shows a color magnitude dependence, so that additional color correction should be applied for an accurate calibration of the TRGB.

We introduce the $Q T$ magnitude, a quadratic form of the TRGB magnitude corrected for the color dependence of the F814W (or I) magnitude TRGB. The $Q T$ is expressed by

$$
Q T=\mathrm{F} 814 \mathrm{~W}_{0}-\alpha(\text { Color }-\gamma)^{2}-\beta(\text { Color }-\gamma)
$$

where $\alpha=0.159 \pm 0.010, \beta=-0.047 \pm 0.020$, and $\gamma=1.1$ in the F606W - F814W filter combination in ACS/WFC. Distance modulus is obtained as follows:

$$
(m-M)_{0}=Q T_{R G B}+M_{Q T, T R G B}
$$

where $M_{Q T, T R G B}$ is an absolute magnitude of the TRGB in the $Q T$ system. This TRGB calibration is called hereafter the $Q T$ calibration. Values of $M_{F 814 W, T R G B}$ and $M_{Q T, T R G B}$ will be addressed in the next Section.

\section{ZERO-POINT CALIBRATION}

\subsection{NGC 4258 as a Distance Anchor}

NGC 4258 is known to be a useful distance anchor because it hosts a water megamaser, which can be used as a powerful and precise geometric distance indicator. With monitoring observations of the nuclear water megamaser sources in NGC 4258, several geometric distance estimates were presented: Greenhill et al. (1995); Mivoshi et al. (1995); Herrnstein et al. (1999); Humphreys et al. (2005); Argon et al. (2007); Humphrevs et al. (2008, 2013); Riess et al. (2016). Humphreys et al. (2013) reported a distance value of $d=7.60 \pm 0.17_{r} \pm 0.15_{s} \mathrm{Mpc}$ $\left((m-M)_{0}=29.404 \pm 0.049_{r} \pm 0.043_{s}\right)$, where errors with $r$ and $s$ represent random errors and systematic errors, respectively. It is slightly larger than the old value given by Herrnstein et al. (1999) $\left(d=7.2 \pm 0.2_{r} \pm 0.5_{s}\right.$ $\mathrm{Mpc}$ ), which is similar to the value used in Riess et al. (2011, 2012) $(d=7.28 \pm 0.22 \mathrm{Mpc})$. Most recently, Riess et al. (2016) provided a similar value with smaller systematic uncertainty, $\mathrm{d}=7.54 \pm 0.17_{r} \pm 0.10_{s} \mathrm{Mpc}$ $\left((m-M)_{0}=29.387 \pm 0.049_{r} \pm 0.029_{s}\right)$. They performed a significantly larger MCMC analysis than Humphreys et al. (2013), reducing the systematic uncertainty by a factor of two. We adopted the most recent distance estimate given by Riess et al. (2016) for the zero-point calibration of the TRGB in this study.

Mager et al. (2008) reported a TRGB distance to NGC 4258 based on photometry of the same HST images as used in this study. They carried out PSF photometry using two software tools, DOLPHOT (Dolphin 2000) and DAOPHOT, and derived a TRGB magnitude, $T_{R G B}=$ $25.24 \pm 0.04$ from DOLPHOT photometry. It is noted that they found $\sim 0.04$ mag difference in the TRGB magnitude between the two data reduction methods.

We obtained a value for the TRGB magnitude in the QT system for NGC 4258 from photometry of ACS image data that we used for the color dependence calibration (Section 2). We also performed a test similar to the Mager et al. (2008)'s. We performed point source photometry based on five different techniques using different softwares and different image types: 1) DAOPHOT/ALLFRAME run on charge transfer efficiency (CTE) corrected drizzled images (indicated by _drc.fits), 2) IRAF/DAOPHOT run on the same_drc.fits images, 3) DAOPHOT/ALLFRAME run on CTE corrected and flat fielded images (indicated by flc.fits), 4) DOLPHOT run on the same _flc.fits images, and 5) DOLPHOT run on CTE uncorrected and flat fielded images (indicated by _flt.fits). We used PSF images we derived using isolated bright stars (for DAOPHOT/ALLFRAME and IRAF/DAOPHOT reductions on drizzled images) or provided by the Tiny Tim (for DOLPHOT reductions). In the case of DAOPHOT/ALLFRAME photometry on individual _flc.fits images, we used PSF images constructed from the ACS images of 47 Tuc (Proposal IDs $=10737$ for F555W and 10101 for F814W filters), because the number of bright stars in a single _flc.fits image of NGC 4258 is only a few. A single pass sequence of DAOFINDPHOT-ALLSTAR/ALLFRAME routines was applied for the IRAF/DAOPHOT and ALLFRAME reductions. DOLPHOT reductions were done using the parameter set recommended by the DOLPHOT/ACS User's guide (version 2.0). We set the ACSuseCTE $=0$ (CTE uncorrection mode) for _flc.fits and 1 (CTE correction mode) 

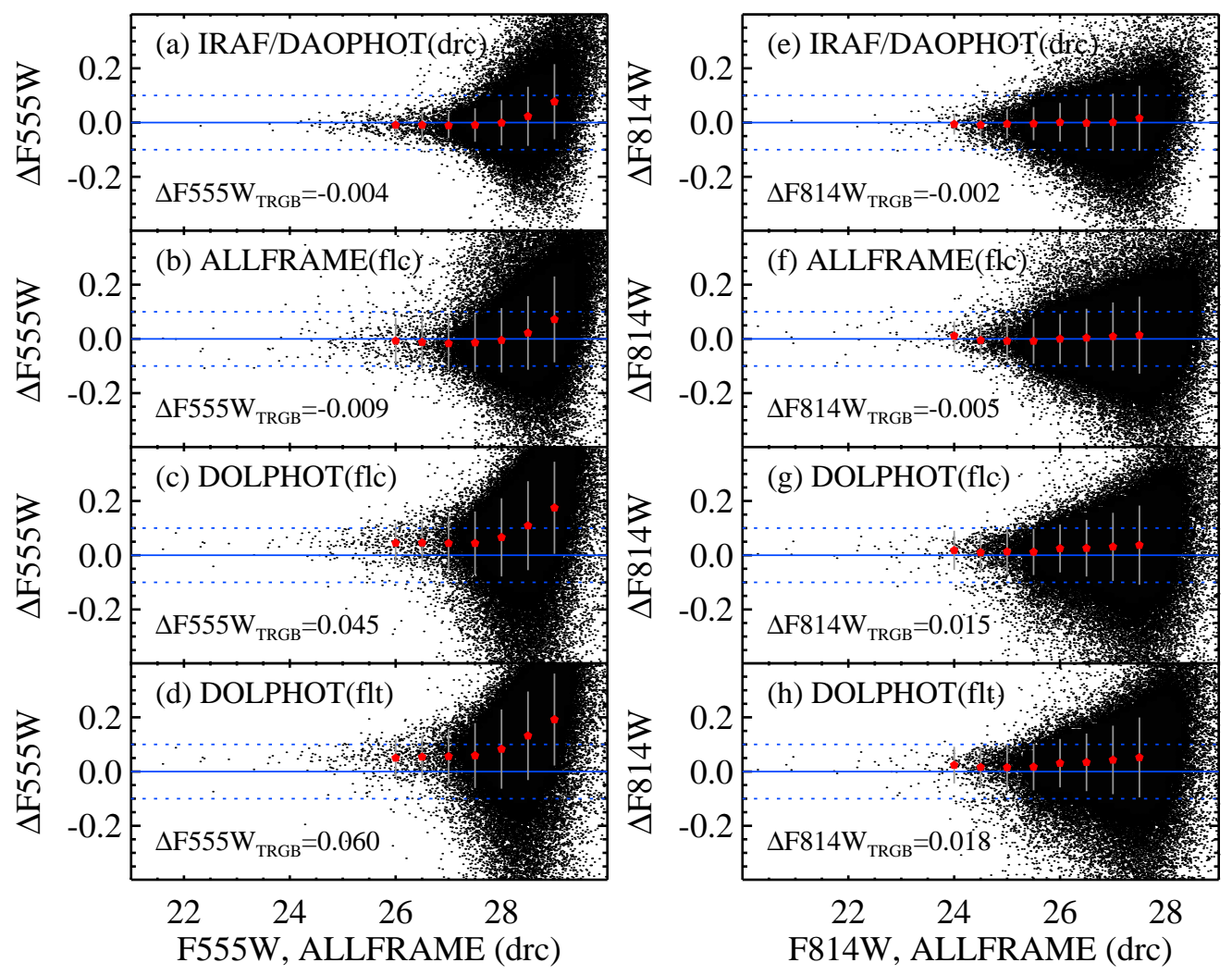

Fig. 6.- (a-d) Comparisons of PSF magnitudes in F555W band between ALLFRAME on drc reduction and IRAF/DAOPHOT on drc (a), ALLFRAME on flc (b), DOLPHOT on flc (c), and DOLPHOT on flt (d) reductions of NGC 4258. Red dots and error bars represent the mean magnitude offset and its standard deviation, respectively. Mean magnitude offsets at the TRGB level $($ F555W $\sim 28.0)$ are labeled in each panel. (e-h) Same as (a-d), except for F814W band. Note the mean magnitude offsets at the TRGB level (F814W $\sim 25.3)$ are very small, $\Delta \mathrm{F} 814 \mathrm{~W} \lesssim 0.02$ mag.

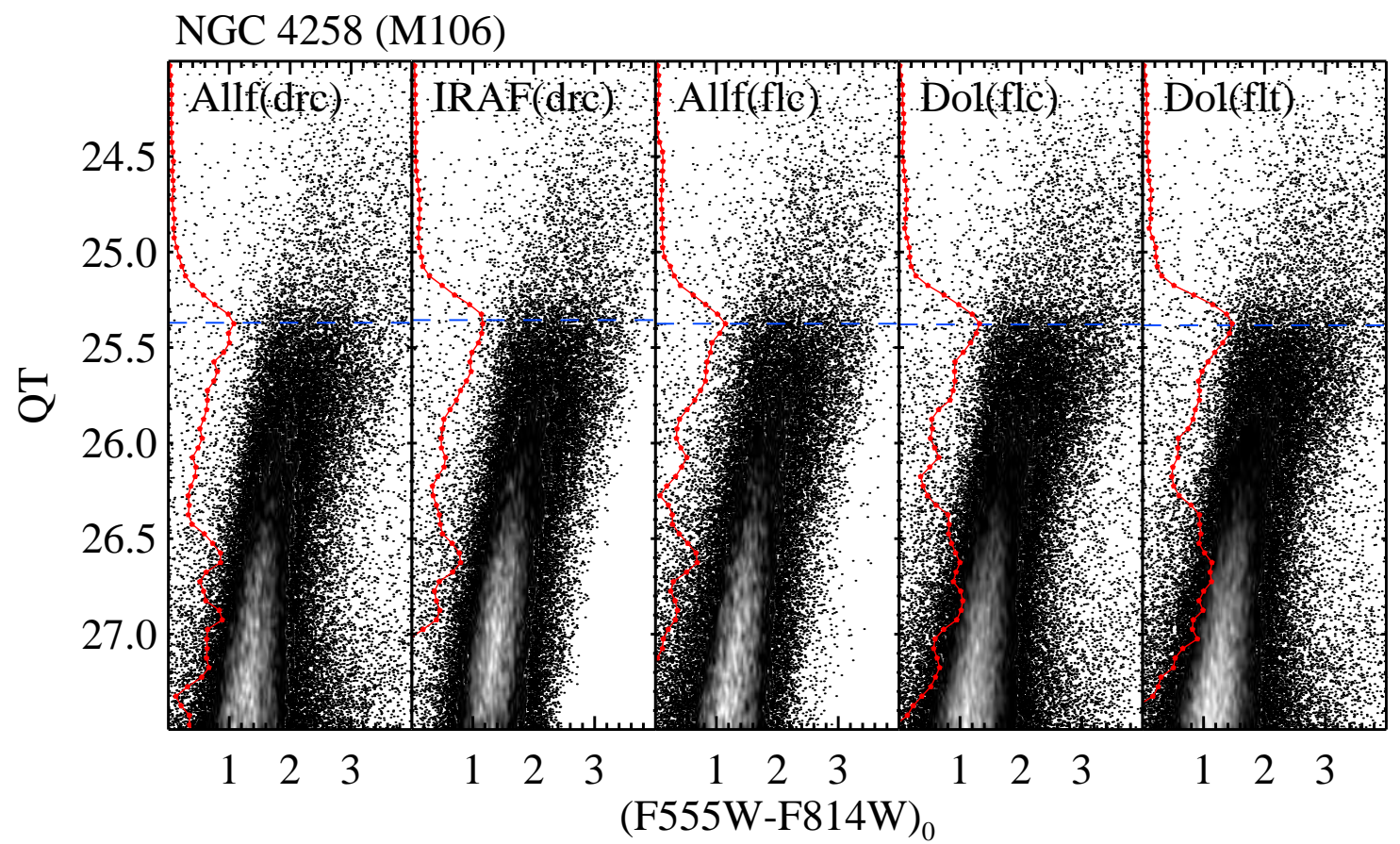

FIG. 7.- QT - (F555W - F814W $)_{0}$ CMDs of NGC 4258 from five different reduction methods : ALLFRAME on drc, IRAF/DAOPHOT on drc, ALLFRAME on flc, DOLPHOT on flc, and DOLPHOT on flt (from left to right). Edge detection responses are shown by the solid lines. Note that the estimated TRGB magnitudes (dashed lines) agree very well. 
for _flt.fits in DOLPHOT reductions.

Figure 6] displays the magnitude differences between the results of the ALLFRAME (drc) reduction and those of other reductions. Comparisons of F555W band magnitudes (left panels) show small offsets. Mean offsets for the bright stars with $\mathrm{F} 555 \mathrm{~W} \lesssim 27.0$ are smaller than 0.05 mag. At the TRGB level, F555W $\sim 27.8$ mag, mean offsets are estimated to be $-0.004,-0.009,0.045$, and $0.060 \mathrm{mag}$ for the IRAF/DAOPHOT (drc), ALLFRAME (flc), DOLPHOT (flc), and DOLPHOT (flt), respectively. Thus, three DAOPHOT reductions (ALLFRAME (drc), IRAF/DAOPHOT (drc), and ALLFRAME (flc)) agree well within $0.01 \mathrm{mag}$, but DOLPHOT reductions show $\sim 0.05 \mathrm{mag}$ differences. Faint stars with F555W $\gtrsim 28.0$ mag yield larger offsets, which increase as a function of magnitude. The origin of these magnitude differences is unclear. Although non-negligible offsets are detected at the faint levels $(F 555 W \gtrsim 28.0)$, most of the RGB stars we used in the TRGB detection of NGC 4258 are brighter than $\mathrm{F} 555 \mathrm{~W} \sim 28.0$, where mean offsets are small enough. Moreover, $F 555 \mathrm{~W}$ band magnitudes are only used in the estimation of the TRGB color, which affects little in the $Q T$ magnitude estimation. Comparisons of F814W band magnitudes show much better agreements. All five reductions agree well within $0.02 \mathrm{mag}$ from the bright to the faint magnitude range $(21.0 \lesssim \mathrm{F} 814 \mathrm{~W} \lesssim 27.5 \mathrm{mag})$.

In Figure 7 we compare $Q T-(\mathrm{F} 555 \mathrm{~W}-\mathrm{F} 814 \mathrm{~W})_{0}$ CMDs from five different reductions. All five CMDs show strong edge detection responses at $Q T \sim 27.4$, which represents clearly a TRGB. ALLFRAME reductions (drc and flc) show sharper responses at $Q T \sim$ 27.4 than that of IRAF/DAOPHOT (drc). Precise TRGB magnitudes and uncertainties are obtained using the bootstrap method as done in Jang \& Lee (2015): $Q T_{R G B}=25.370 \pm 0.023,25.356 \pm 0.053,25.375 \pm$ $0.021,25.379 \pm 0.018,25.283 \pm 0.019 \mathrm{mag}$ for ALLFRAME(drc), IRAF/DAOPHOT (drc), ALLFRAME (flc), DOLPHOT (flc), and DOLPHOT(flt), respectively. These estimations yield a mean of $Q T_{R G B}=25.373 \mathrm{mag}$, median of $Q T_{R G B}=25.375 \mathrm{mag}$, and standard deviation of $0.010 \mathrm{mag}$, which is very small. Thus our TRGB estimation is robust. Software-dependent uncertainties in the TRGB detection are not significant. We use the TRGB magnitude from the ALLFRAME (drc) reduction, $Q T_{R G B}=25.370 \pm 0.023$ in the following analysis.

We also determine a color dependence uncorrected TRGB magnitude, F814W $\mathrm{W}_{0, T R G B}$. Figure 8 shows $\mathrm{F}_{814} \mathrm{~W}_{0}-(\mathrm{F} 555 \mathrm{~W}-\mathrm{F} 814 \mathrm{~W})_{0}$ CMD of NGC 4258 from the ALLFRAME (drc) reduction. We selected the blue RGB stars in the shaded region where color dependence of the TRGB is less severe $\left((\mathrm{F} 555 \mathrm{~W}-\mathrm{F} 814 \mathrm{~W})_{0} \leq 2.1\right)$ and plotted their edge detection response by a solid line. The edge detection response is relatively noisy compared to that from the $Q T \mathrm{CMD}$, but still shows a major peak at $\mathrm{F} 814 \mathrm{~W}_{0}=25.357 \pm 0.031 \mathrm{mag}$, which is the TRGB

From the TRGB magnitudes derived in this study $\left(Q T=25.370 \pm 0.023 \mathrm{mag}\right.$ and $\mathrm{F} 814 \mathrm{~W}_{0}=25.357 \pm 0.031$ mag) and the distance modulus to NGC 4258 given by Riess et al. (2016), we determine the zero-points of the TRGB: $M_{\mathrm{QT}}$ TRGB $=-4.023 \pm 0.073 \mathrm{mag}$ for the $Q T$ calibration and $M_{\mathrm{F} 814 \mathrm{~W}, \mathrm{TRGB}}=-4.036 \pm 0.074 \mathrm{mag}$ for the blue $I$ calibration. Their uncertainties, $0.073 \mathrm{mag}$ and $0.074 \mathrm{mag}$ are obtained from the quadratic sums of indi-

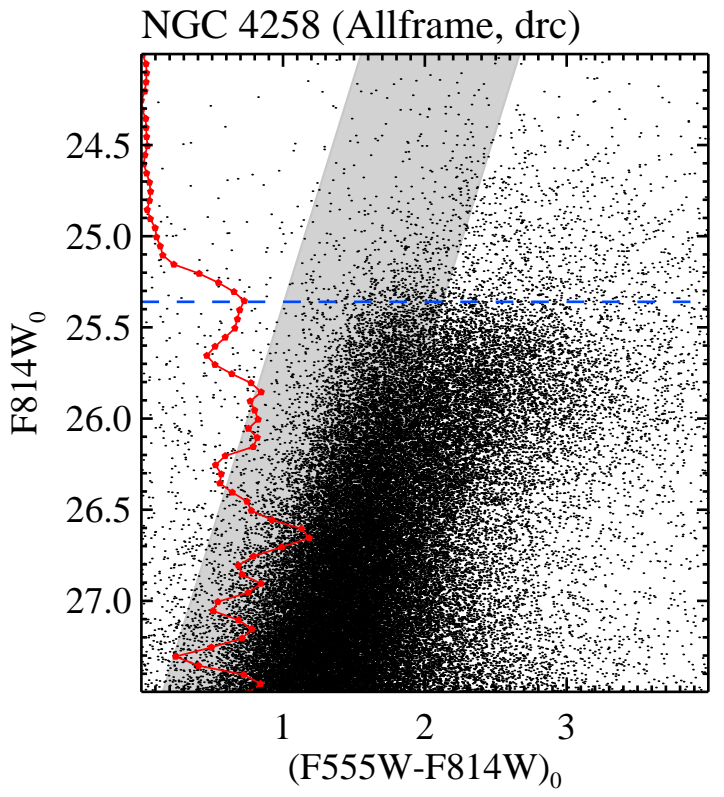

FIG. 8.- F814W 0 -(F555W-F814W $)_{0}$ CMD of NGC 4258 from the ALLFRAME (drc) reduction. An edge detection response for resolved stars in the shaded region is shown by a solid line. The TRGB is estimated to be at $\mathrm{F} 814 \mathrm{~W}_{0}=25.36 \mathrm{mag}$ (dashed line).

TABLE 4

Error Budget for the $Q T$ Calibration of the TRGB in the STANDARD $V I$ SYSTEM

\begin{tabular}{lcccc}
\hline \multicolumn{1}{c}{ Error source } & \multicolumn{2}{c}{$Q T$ calibration } & \multicolumn{2}{c}{ Blue $I$ calibration } \\
& NGC 4258 & LMC & NGC 4258 & LMC \\
\hline TRGB detection & 0.023 & 0.020 & 0.031 & 0.042 \\
Extinction & 0.003 & 0.07 & 0.003 & 0.07 \\
Photometric zero-point & 0.02 & 0.03 & 0.02 & 0.03 \\
Color transformation ${ }^{a}$ & 0.005 & $\ldots$ & $\ldots$ & $\ldots$ \\
F814W to I transformation & 0.03 & $\ldots$ & 0.03 & $\ldots$ \\
$Q T$ transformation & 0.014 & 0.014 & $\ldots$ & $\ldots$ \\
Intermediate-age population & $\ldots$ & 0.02 & $\ldots$ & 0.02 \\
Distance anchor & 0.057 & 0.049 & 0.057 & 0.049 \\
\hline Total & 0.073 & 0.096 & 0.074 & 0.102 \\
& & & &
\end{tabular}

F555W-F814W to F606W-F814W transformation

vidual uncertainties as summarized in Table 4. TRGB detection errors $(0.023 \mathrm{mag}$ and $0.031 \mathrm{mag})$, an extinction correction error $(0.003 \mathrm{mag}, 10 \%$ of $\mathrm{F} 814 \mathrm{~W}$ band extinction), a photometric zero-point error (0.020 mag), a color transformation error $(0.005 \mathrm{mag})$, an F814W to QT transformation error (0.014 mag), and an NGC 4258 distance error $(0.057 \mathrm{mag})$. When we use the $Q T$ calibration in the standard VI system, a photometric transformation error $(0.03 \mathrm{mag})$ is added. Thus systematic uncertainties are dominated by the NGC 4258 distance error.

\subsection{The Large Magellanic Cloud as a Distance Anchor}

The LMC has been often used as a distance anchor for extragalactic distance indicators (Freedman \& Madore 2010; de Grijs et al. 2014). Its distance has long been controversial from $(m-M)_{0}=18.1$ to 18.8 , and it appeared to settle down with a value of $(m-M)_{0} \sim$ $18.50 \pm 0.10$, after the results of the Hubble Key Project (Freedman et al. 2001) were published. However, it has 


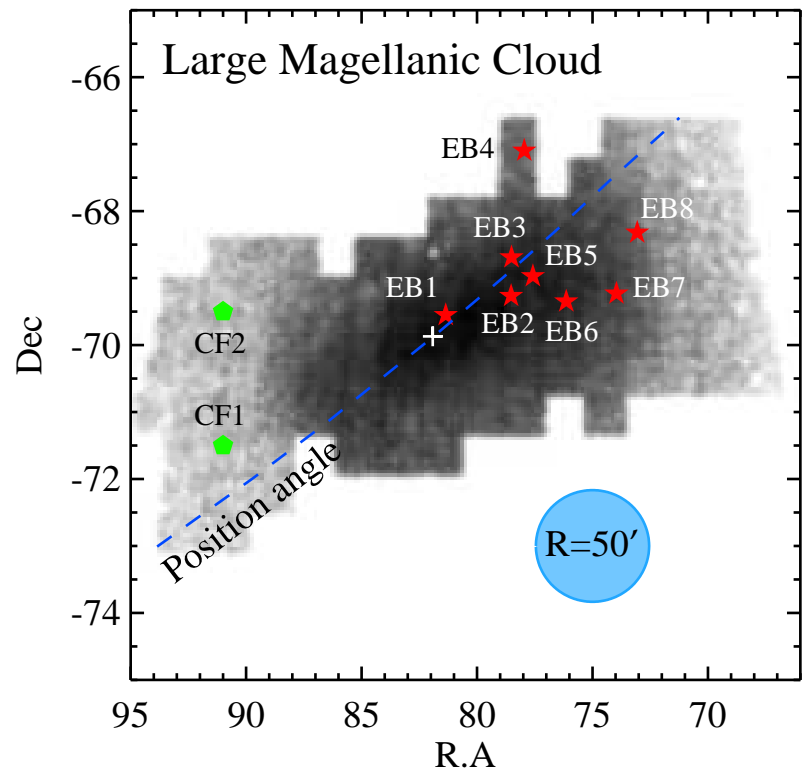

FIG. 9.- Identification of eight eclipsing binary stars (starlets) used in Pietrzyński et al. (2013), overlayed on a number density map of RGB stars in the LMC. Two control fields used in this study are marked by green pentagons. We investigated the TRGB magnitude of the LMC using the stars at $R \leq 50^{\prime}$ region of each eclipsing binary star and each control field.

been suspected that the tight correlation of the LMC distance estimates might have been affected by the publication bias or the bandwagon effect (Schaefer 2008; de Griis et al. 2014). This problem can be resolved with more accurate distance estimates for the LMC in the future.

Recently Freedman et al. (2012) presented an updated distance estimate, $(m-M)_{0}=18.477 \pm 0.011_{r} \pm$ $0.033_{s}$, accurate to $1.7 \%$, from the analysis of Spitzer $3.6 \mu \mathrm{m}$ photometry for 10 Cepheids in the Milky Way and 80 Cepheids in the LMC. On the other hand, Pietrzyński et al. (2013) analyzed eight eclipsing binary stars using the OGLE photometry, and yielded a distance value with a total uncertainty of $2.2 \%:(m-M)_{0}=$ $18.494 \pm 0.008_{r} \pm 0.048_{s}\left(d=49.97 \pm 0.19_{r} \pm 0.11_{s} \mathrm{kpc}\right)$. These two recent estimates agree very well. We adopted the distance estimate by Pietrzyński et al. (2013) for the zero-point calibration of the TRGB, because we use the OGLE photometry of the LMC for the analysis of the TRGB in this study as follows.

We measured the TRGB magnitude of the LMC using the VI photometric catalog from the OGLE-III shallow survey (Ulaczvk et al. 2012). This catalog was made using the same telescope and the same CCD camera as used for the analysis of Pietrzyński et al. (2013). It offers benefits that any instrument-dependent uncertainties, which are hard to measure, can be ruled out in the zero point calibration of the TRGB. Figure 9 shows identifications of eight eclipsing binary stars used in Pietrzyński et al. (2013) (starlets) overlayed on a spatial number density map of bright RGB stars $(1.2<V-I \leq 2.0$ and $14.4<I \leq 16.0)$ in the OGLE-III catalog. All eight binary stars are located on the western side from the LMC center (white cross). It is known that the distance measurement can be affected by the geometry and the depth of the LMC. For this reason, Pietrzyński et al.

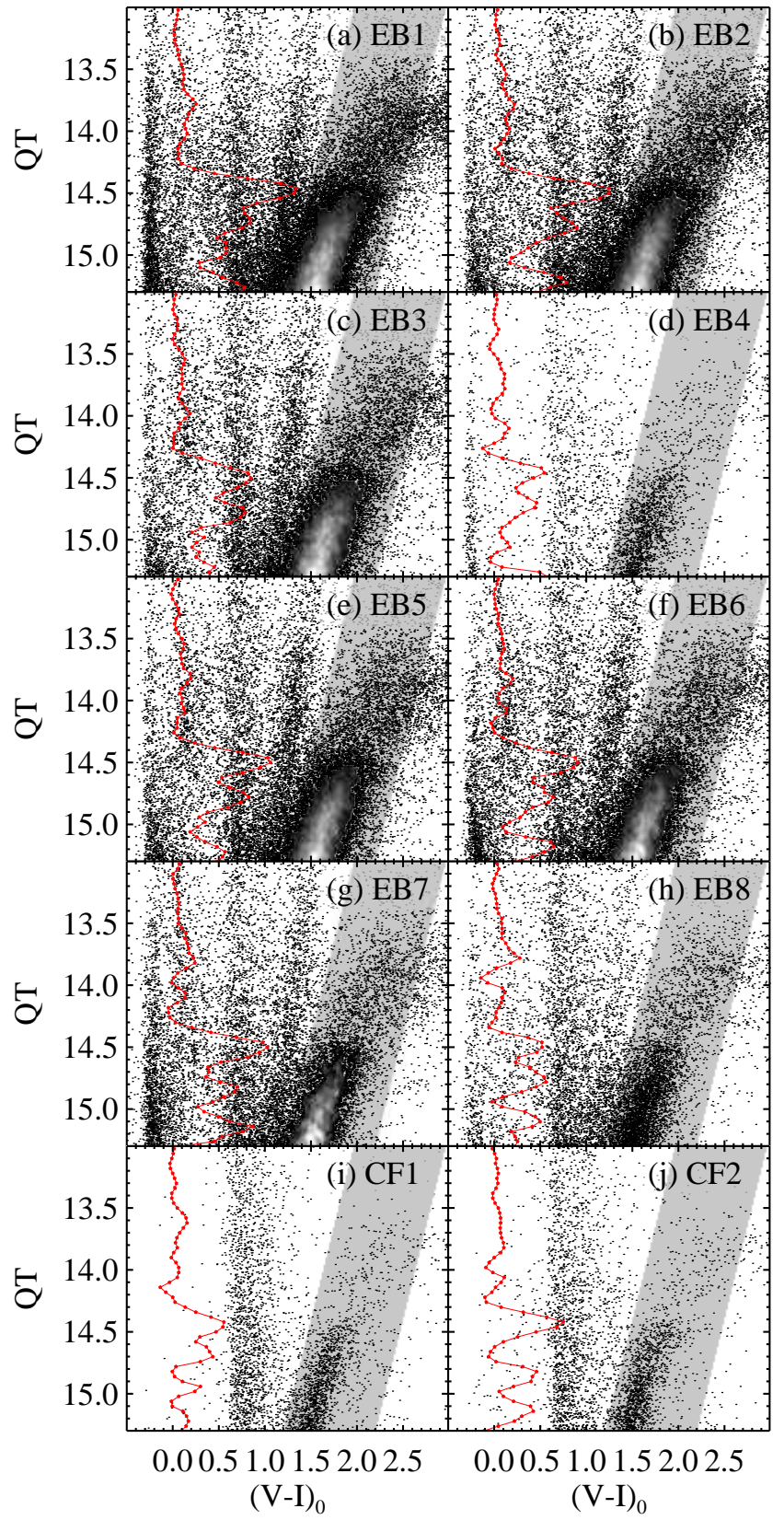

FIG. 10.- $Q T-(V-I)_{0}$ CMDs of resolved stars in $R \leq 50^{\prime}$ regions of eclipsing binary stars (a-h) and control fields (i-j). Shaded regions indicate the area we used to sample the RGB stars for the TRGB analysis. Edge detection responses are shown by the solid lines.

(2013) applied additional geometry correction to their distance estimation. However, they concluded that the effect of this correction to the distance measurement is negligible. For the analysis of the TRGB for the LMC we selected eight fields located around the eclipsing binary stars used in Pietrzyński et al. (2013), and used the stars at $R \leq 50^{\prime}$ region around each eclipsing binary star. We investigated also two additional fields, control field 1 and 2 (pentagons), covering the eastern side of the LMC as a reference.

In Figure 10, we plotted the extinction corrected $Q T-(V-I)_{0}$ CMDs of eight fields around eclipsing binary stars $(a-h)$ and two control fields $(i, j)$. The extinc- 
TABLE 5

A Summary of the TRGB Magnitudes of the LMC

\begin{tabular}{|c|c|c|c|c|c|c|}
\hline \multirow[t]{2}{*}{ Field } & \multirow[t]{2}{*}{ R.A. } & \multirow[t]{2}{*}{ Decl } & \multicolumn{2}{|c|}{$Q T^{a}$ magnitude } & \multicolumn{2}{|c|}{$I^{b}$ magnitude } \\
\hline & & & (Measured) & (Distortion corrected) & (Measured) & (Distortion corrected) \\
\hline EB1 & 052525.550 & -693304.39 & $14.485 \pm 0.010$ & $14.489 \pm 0.010$ & $14.521 \pm 0.035$ & $14.525 \pm 0.035$ \\
\hline EB2 & 051405.952 & -691556.83 & $14.486 \pm 0.011$ & $14.482 \pm 0.011$ & $14.522 \pm 0.017$ & $14.518 \pm 0.017$ \\
\hline EB4 & 051149.458 & -670545.19 & $14.459 \pm 0.012$ & $14.485 \pm 0.012$ & $14.475 \pm 0.013$ & $14.501 \pm 0.013$ \\
\hline EB5 & 051019.650 & -685812.00 & $14.502 \pm 0.014$ & $14.498 \pm 0.014$ & $14.480 \pm 0.023$ & $14.476 \pm 0.023$ \\
\hline EB6 & 050432.882 & -692050.99 & $14.505 \pm 0.015$ & $14.489 \pm 0.015$ & $14.580 \pm 0.015$ & $14.564 \pm 0.015$ \\
\hline EB7 & 045551.491 & -691347.99 & $14.511 \pm 0.016$ & $14.487 \pm 0.016$ & $14.569 \pm 0.016$ & $14.565 \pm 0.016$ \\
\hline $\mathrm{CF} 2$ & 060400.000 & -693000.00 & $14.425 \pm 0.015$ & $14.472 \pm 0.015$ & $14.472 \pm 0.021$ & $14.519 \pm 0.021$ \\
\hline \multicolumn{3}{|c|}{ Weighted mean of EB1-8 and CF1-2 } & $14.485 \pm 0.004(0.030)^{c}$ & $14.489 \pm 0.004(0.011)$ & $14.515 \pm 0.006(0.042)$ & $14.522 \pm 0.006(0.036)$ \\
\hline \multicolumn{3}{|c|}{ Weighted mean of CF1-2 } & $14.432 \pm 0.013(0.025)$ & $14.475 \pm 0.013(0.009)$ & $14.470 \pm 0.016(0.003)$ & $14.504 \pm 0.016(0.026)$ \\
\hline \multicolumn{3}{|c|}{ Weighted mean of EB1-8 } & $14.491 \pm 0.004(0.020)$ & $14.490 \pm 0.004(0.009)$ & $14.523 \pm 0.007(0.042)$ & $14.524 \pm 0.007(0.039)$ \\
\hline
\end{tabular}

${ }^{a}$ A wide color range of the TRGB with $1.5 \leq(V-I)_{0, T R G B} \leq 2.5$ was used.

b The blue color range of the TRGB with $1.5 \leq(V-I)_{0, T R G B} \leq 1.9$ was used.

${ }^{c}$ Denotes standard deviation.

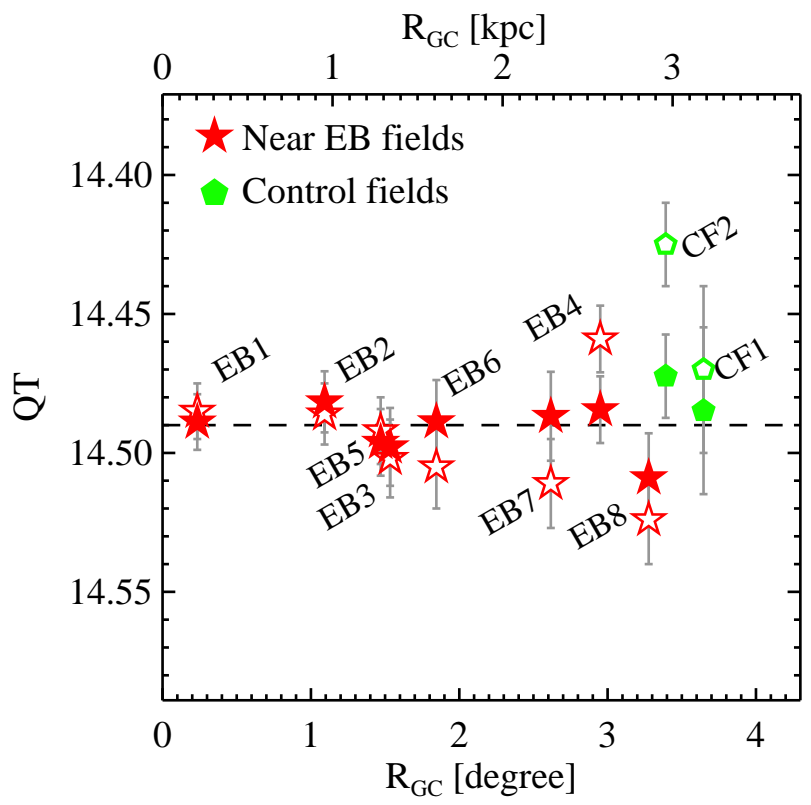

FIG. 11. - A comparison of the TRGB magnitudes from the fields around eclipsing binary stars (starlets) and from the control fields (pentagons) vs. galactocentric radius of the LMC. The open and filled symbols denote the TRGB magnitudes before and after geometric distortion correction, respectively. The mean TRGB magnitude of eight eclipsing binary fields, corrected for the geometric distortion (filled stars), is estimated to be $Q T=14.90 \pm 0.004$ (dashed line).

tion correction was done using the extinction map of the LMC given by Haschke et al. (2011), who estimated the extinction values from the mean color of red clump stars in the OGLE-III photometry. It is noted that we used the same extinction map as applied in Pietrzyński et al. (2013). All ten CMDs show various populations of stars including young main sequence stars (a vertical feature at $\left.(V-I)_{0} \sim 0.2\right)$, blue and red helium burning stars (vertical and slanted features at $(V-I)_{0} \sim 0.1$ and 1.2 , respectively), old RGB stars (a dominant broad slanted feature at $\left.(V-I)_{0} \sim 1.8\right)$ and AGB stars (a broad slanted feature above the RGB) in the LMC. The Milky Way foreground stars are also seen (vertical feature at $(V-I)_{0} \sim 0.7$ ).
We measured the TRGB using the stars located in the shaded regions in Figure 10, which were designed to avoid young stellar populations and to sample RGB stars as many as possible. We plotted the edge detection responses as curved lines in each panel. All ten CMDs show strong peaks at $Q T \sim 14.5 \mathrm{mag}$. We obtained quantitative values of the TRGB magnitudes and corresponding errors from the bootstrap resampling of ten thousand simulations. We also obtained the color dependence uncorrected TRGB magnitude, $I_{0, T R G B}$, by applying the same TRGB analysis to $I-(V-I)_{0}$ CMDs of the ten fields. Only the blue TRGB stars $\left(1.5 \leq(V-I)_{0} \leq 1.9\right)$ showing a weak color dependence of the TRGB were used for the detection. A summary of the $Q T$ and $I$ magnitudes of the TRGB in each field of the LMC is listed in Table 5 .

Figure 11 shows a comparison of the TRGB magnitudes in the $Q T$ system of eight eclipsing binary fields (starlets) and two control fields (pentagons) in the LMC. We corrected geometric distortion of the LMC by assuming the LMC disk with an inclination angle of $28^{\circ}$ and a position angle of $128^{\circ}$, as assumed in Pietrzyński et al. (2013). We plotted the TRGB magnitudes before and after correction of geometric distortion in Figure 11 by open and filled symbols, respectively. The TRGB magnitudes are ranging from $Q T \approx 14.425$ to 14.524 mag. A weighted mean value of the TRGB magnitudes of all ten fields is $Q T=14.485 \pm 0.004 \mathrm{mag}$ with a standard deviation of $0.030 \mathrm{mag}$. Similar values of $Q T=14.491 \pm 0.004$ mag and $Q T=14.432 \pm 0.013 \mathrm{mag}$ are derived from the eight eclipsing binary fields and two control fields, respectively. Distortion correction contributes a very small change to the TRGB magnitudes: $0.004 \mathrm{mag}$ for all ten fields, $0.043 \mathrm{mag}$ for the two control fields, and 0.001 mag for the eight eclipsing binary fields. Similarly, distortion correction changes $I$-band TRGB magnitude of $0.007 \mathrm{mag}$ for all ten fields, $0.034 \mathrm{mag}$ for the two control fields, and $0.001 \mathrm{mag}$ for the eight eclipsing binary fields (Table 5). Thus geometric distortion of the LMC is not significant in the TRGB estimation in this study.

The eight eclipsing binary fields used in this study cover the bar and disk of the LMC, so that we need to check the effect of composite stellar populations in 


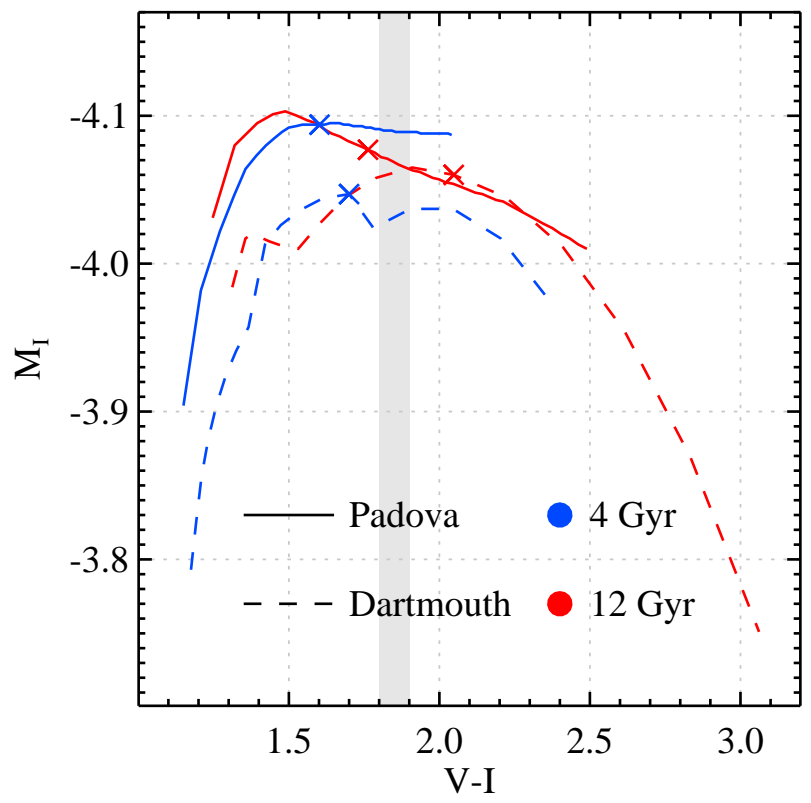

FIG. 12.- The TRGB for 4 Gyr (blue lines) and 12 Gyr (red lines) populations from the Padova (Girardi et al. 2000; Marigo et al. 2013) (solid lines) and the Dartmouth (Dotter et al. 2008) (dashed lines) models. Adopted metallicity range is $-2.3<$ $[\mathrm{Fe} / \mathrm{H}] \leq-0.5$. $[\mathrm{Fe} / \mathrm{H}]=-1.0$ points are marked by crosses. A grey vertical region at $(V-I)=1.9$ indicates the mean TRGB color of the LMC. Note that the TRGB for 4 Gyr Padova model at $(V-I)=1.9$ is $\sim 0.03$ mag brighter than that of 12 Gyr model, whereas, those of Dartmouth models is $\sim 0.03$ mag fainter.

the TRGB estimation. Salaris \& Girardi (2005) conducted an analysis of artificial synthesis populations based on star-formation history of the LMC derived by Holtzman et al. (1999) and showed that intermediateage stars ( $\sim 4$ Gyr) can contaminate the RGB, resulting in wrong measurement of metallicity from colors by $\mathrm{d}[\mathrm{Fe} / \mathrm{H}]=0.5$ dex. They also pointed out that the metallicity difference of 0.5 dex may lead to 0.1 mag difference for the bolometric magnitude and corresponding $I$-band magnitude of the TRGB, when they use the TRGB calibration given in Lee et al. (1993), $M_{b o l, T R G B}$ $=-0.19[\mathrm{Fe} / \mathrm{H}]-3.81$ mag. However, they didn't show how much the TRGB magnitudes vary with different ages from their artificial CMD.

We compared the TRGB magnitudes for the old (12 Gyr) and the intermediate age (4 Gyr) populations using stellar isochrones provided by the Padova (Girardi et al. 2000; Marigo et al. 2013) and Dartmouth (Dotter et al. 2008) groups and plotted them in Figure 12. At the mean TRGB color of the LMC, $(V-I)=1.8 \sim 1.9$, measured from our study, the intermediate age population from the Padova group (blue solid line) shows 0.02 mag brighter magnitude than that of the old population (red solid line). However, those of the Dartmouth group show an inverse trend: the intermediate age population is $0.03 \mathrm{mag}$ fainter than that of the old population. This result implies that the the effect of the intermediate age population for the TRGB measurement is probably smaller than 0.03 mag.

Two control fields used in this study, CF1 and CF2 shown in Figure 10 ( $\mathrm{i}$ and $\mathrm{j}$ ) are located far from the LMC bar, showing no evidence of young and intermediate age population in the CMD. They are dominated by old halo stars. Measured $Q T$ magnitudes of these two control fields are, on average, not different from those of other eight fields in the LMC disk and bar. The mean differences between two control fields and the other eight fields are $0.015 \mathrm{mag}$ in $Q T$ and $0.020 \mathrm{mag}$ in $I$ after geometric correction. From these results, we quote $0.02 \mathrm{mag}$ as a systematic uncertainty for the mixture population of the LMC.

We adopted the distortion corrected mean TRGB magnitude of eight eclipsing binary fields, $Q T=14.490 \mathrm{mag}$ and $I=14.524$, as mean values for TRGB magnitudes of the LMC. Conservative errors, $0.020 \mathrm{mag}$ and 0.042 mag, which are standard deviations of these fields before distortion correction, is adopted as measurement errors. Finally we obtain the zero-points of the TRGB: $M_{Q T, T R G B}=-4.004 \pm 0.096 \mathrm{mag}$ for the $Q T$ calibration and $M_{I, T R G B}=-3.970 \pm 0.102 \mathrm{mag}$ for the blue $I$ calibration. Quoted uncertainties, $0.096 \mathrm{mag}$ and $0.102 \mathrm{mag}$, are derived as summarized in Table 4. TRGB detection errors $(0.020 \mathrm{mag}$ and $0.042 \mathrm{mag})$, an extinction correction error (0.07 mag (Haschke et al. 2011; Górski et al. 2016)), a photometric zero-point error (0.03 mag), the QT transformation error (0.014 mag), and the LMC distance error $(0.049 \mathrm{mag})$. The systematic uncertainty is dominated by the extinction error and the LMC distance error.

\section{SUMMARY OF THE REVISED TRGB CALIBRATION}

In Table 6. we summarize the two revised TRGB calibrations, the $Q T$ and the blue $I$ calibrations, in several photometric systems. Color dependence correction terms $(\alpha, \beta$, and $\gamma)$ in these photometric systems were determined by applying the photometric transformation as described in Section 2.4. The same method was applied to obtain the zero-point values in each photometric system. The zero-point uncertainties associated with the photometric transformation from F $814 \mathrm{~W}$ in ACS to other systems are estimated to be 0.03 mag for the $I$, 0.01 mag for both F555W-F814W and F606W-F814W combinations of $\mathrm{F} 814 \mathrm{~W}$ in WFC3, $0.015 \mathrm{mag}$ for F $555 \mathrm{~W}$ F814W conbination of F814W in WFPC2, and 0.106 mag for $\mathrm{F} 606 \mathrm{~W}-\mathrm{F} 814 \mathrm{~W}$ combination of $\mathrm{F} 814 \mathrm{~W}$ in WFPC2 (Sirianni et al. 2005; Jang \& Lee 2015).

Zero-points from NGC 4258 and the LMC shows a good agreement. A weighted mean of the zero-points from NGC 4258 and the LMC yields $M_{Q T, T R G B}=$ $-4.016 \pm 0.058$ and $M_{I, T R G B}=-4.013 \pm 0.060$ in $I$-band. These values are similar to the values given in previous calibrations, $M_{I T R G B}=-4.05 \pm 0.12$ (Bellazzini et al. 2001, 2004; Rizzi et al.|2007; (Madore et al.|2009), but its quoted error is two times smaller.

\section{DISCUSSION}

\subsection{Comparison with Previous TRGB Calibrations}

There have been several studies of the TRGB calibration including both color-magnitude dependence and zero-point calibrations as summarized in Table 7 Da Costa \& Armandroff (1990) provided the bolometric magnitude and bolometric correction of the TRGB as a function of metallicity and color, based on VI photometry of six globular clusters in the Milky Way. With these relations, Lee et al. (1993) estimated the absolute 
TABLE 6

A Summary of the TRGB Calibrations in This Study

\begin{tabular}{|c|c|c|c|c|c|c|c|c|}
\hline \multirow[b]{2}{*}{ System } & \multicolumn{4}{|c|}{$Q T=I(\mathrm{~F} 814 \mathrm{~W})_{0}-\alpha(\text { Color }-\gamma)^{2}-\beta($ Color $-\gamma)$} & \multirow[b]{2}{*}{ Color range } & \multicolumn{3}{|c|}{ Zero-point } \\
\hline & Color & $\alpha$ & $\beta$ & & & NGC 4258 & LMC & $\mathrm{N} 4258+\mathrm{LMC}$ \\
\hline \multicolumn{9}{|l|}{$Q T$ calibration } \\
\hline ACS/WFC & $(\mathrm{F} 606 \mathrm{~W}-\mathrm{F} 814 \mathrm{~W})_{0}$ & $0.159 \pm 0.010$ & $-0.047 \pm 0.020$ & 1.1 & $\ldots$ & $-4.021 \pm 0.067$ & $-4.002 \pm 0.101$ & $-4.015 \pm 0.056$ \\
\hline $\mathrm{ACS} / \mathrm{WFC}$ & $(\mathrm{F} 555 \mathrm{~W}-\mathrm{F} 814 \mathrm{~W})_{0}$ & $0.116 \pm 0.007$ & $-0.043 \pm 0.017$ & 1.6 & $\ldots$ & $-4.017 \pm 0.067$ & $-3.998 \pm 0.101$ & $-4.011 \pm 0.056$ \\
\hline J.C. & $(V-I)_{0}$ & $0.091 \pm 0.006$ & $-0.007 \pm 0.013$ & 1.5 & $\ldots$ & $-4.023 \pm 0.073$ & $-4.004 \pm 0.096$ & $-4.016 \pm 0.058$ \\
\hline WFC3/UVIS & $(\mathrm{F} 606 \mathrm{~W}-\mathrm{F} 814 \mathrm{~W})_{0}$ & $0.150 \pm 0.010$ & $-0.050 \pm 0.023$ & 1.1 & $\ldots$ & $-4.031 \pm 0.068$ & $-4.012 \pm 0.101$ & $-4.025 \pm 0.057$ \\
\hline WFC3/UVIS & $(\mathrm{F} 555 \mathrm{~W}-\mathrm{F} 814 \mathrm{~W})_{0}$ & $0.113 \pm 0.007$ & $-0.048 \pm 0.019$ & 1.6 & $\ldots$ & $-4.026 \pm 0.068$ & $-4.007 \pm 0.101$ & $-4.020 \pm 0.057$ \\
\hline WFPC2 & $(\mathrm{F} 606 \mathrm{~W}-\mathrm{F} 814 \mathrm{~W})_{0}$ & $0.179 \pm 0.012$ & $-0.034 \pm 0.024$ & 1.0 & $\ldots$ & $-3.992 \pm 0.125$ & $-3.973 \pm 0.139$ & $-3.984 \pm 0.121$ \\
\hline WFPC2 & $(\mathrm{F} 555 \mathrm{~W}-\mathrm{F} 814 \mathrm{~W})_{0}$ & $0.123 \pm 0.008$ & $-0.051 \pm 0.020$ & 1.5 & $\ldots$ & $-4.014 \pm 0.069$ & $-3.995 \pm 0.092$ & $-4.007 \pm 0.060$ \\
\hline \multicolumn{5}{|c|}{ Blue I calibration } & & & & \\
\hline $\mathrm{ACS} / \mathrm{WFC}$ & $(\mathrm{F} 606 \mathrm{~W}-\mathrm{F} 814 \mathrm{~W})_{0}$ & $\ldots$ & .. & $\ldots$ & Color $\leq 1.5$ & $-4.034 \pm 0.068$ & $-3.968 \pm 0.106$ & $-4.015 \pm 0.057$ \\
\hline $\mathrm{ACS} / \mathrm{WFC}$ & $(\mathrm{F} 555 \mathrm{~W}-\mathrm{F} 814 \mathrm{~W})_{0}$ & $\ldots$ & $\ldots$ & $\ldots$ & Color $\bar{x} 2.1$ & $-4.030 \pm 0.068$ & $-3.964 \pm 0.106$ & $-4.008 \pm 0.057$ \\
\hline J.C. & $(V-I)_{0}$ & $\cdots$ & $\cdots$ & $\cdots$ & Color $\leq 1.9$ & $-4.036 \pm 0.074$ & $-3.970 \pm 0.102$ & $-4.013 \pm 0.060$ \\
\hline WFC3/UVIS & $(\mathrm{F} 606 \mathrm{~W}-\mathrm{F} 814 \mathrm{~W})_{0}$ & $\ldots$ & $\ldots$ & $\ldots$ & Color $\overline{\leq} 1.5$ & $-4.044 \pm 0.069$ & $-3.978 \pm 0.107$ & $-4.025 \pm 0.058$ \\
\hline WFC3/UVIS & $(\mathrm{F} 555 \mathrm{~W}-\mathrm{F} 814 \mathrm{~W})_{0}$ & $\ldots$ & $\ldots$ & $\ldots$ & Color $\overline{\leq} 2.1$ & $-4.039 \pm 0.069$ & $-3.973 \pm 0.107$ & $-4.020 \pm 0.058$ \\
\hline WFPC2 & $(\mathrm{F} 606 \mathrm{~W}-\mathrm{F} 814 \mathrm{~W})_{0}$ & $\ldots$ & $\ldots$ & $\ldots$ & Color $\leq 1.5$ & $-4.005 \pm 0.126$ & $-3.939 \pm 0.143$ & $-3.976 \pm 0.121$ \\
\hline WFPC2 & $(\mathrm{F} 555 \mathrm{~W}-\mathrm{F} 814 \mathrm{~W})_{0}$ & $\ldots$ & $\ldots$ & $\ldots$ & Color $\leq 1.9$ & $-4.027 \pm 0.069$ & $-3.961 \pm 0.098$ & $-4.005 \pm 0.062$ \\
\hline
\end{tabular}

TABLE 7

A List of the Previous and Revised TrGB Calibrations

\begin{tabular}{|c|c|c|c|}
\hline Reference & Calibration & $\begin{array}{l}\text { Zero-point } \\
M_{I, T R G B} \\
\end{array}$ & Note \\
\hline $\begin{array}{l}\text { Lee et al. (1993) } \\
\text { Salaris \& Cassisi (1997) } \\
\text { Ferrarese et al. }(2000) \\
\text { Ferrarese et al. } 2000) \\
\text { Bellazzini et al. (2001) } \\
\text { Bellazzini et al. (2004) } \\
\text { Rizzi et al. (2007) } \\
\text { Bellazzini (2008) } \\
\text { Tammann et al. (2008) } \\
\text { Madore et al. (2009) } \\
\text { Fusco et al. (2012) } \\
\text { This study }\end{array}$ & $\begin{array}{l}-4.0 \pm 0.1 \\
-3.732+0.588[\mathrm{M} / \mathrm{H}]+0.193[\mathrm{M} / \mathrm{H}]^{2} \\
-4.06 \pm 0.07 \pm 0.13 \\
-3.99 \pm 0.07 \pm 0.13 \\
-3.66+0.48[\mathrm{Fe} / \mathrm{H}]+0.14[\mathrm{Fe} / \mathrm{H}]^{2} \\
-3.629+0.679[\mathrm{M} / \mathrm{H}]+0.258[\mathrm{M} / \mathrm{H}]^{2} \\
-4.05+0.217\left[(V-I)_{0}-1.6\right] \\
-3.939-0.194(V-I)_{0}+0.080(V-I)_{0}^{2} \\
-4.05 \pm 0.02 \pm 0.10 \\
-4.05+0.2\left[(V-I)_{0}-1.5\right] \\
-3.63-0.40 C o l^{a}+0.08 C o l^{2} \\
-4.016+0.091\left((V-I)_{0}-1.5\right)^{2}-0.007\left((V-I)_{0}-1.5\right)\end{array}$ & $\begin{array}{l}-4.0 \pm 0.1 \\
-4.16 \\
-4.06 \pm 0.07 \pm 0.13 \\
-3.99 \pm 0.07 \pm 0.13 \\
-4.07 \pm 0.12 \\
-4.07 \pm 0.12 \\
-4.07 \\
-4.05 \\
-4.05 \pm 0.02 \pm 0.10 \\
-4.05 \\
-4.13 \pm 0.01 \\
-4.015 \pm 0.059\end{array}$ & $\begin{array}{l}\text { MW GCs } \\
-2.35 \leq[\mathrm{M} / \mathrm{H}] \leq-0.57 \\
\text { Cepheids, } \gamma=0.00 \\
\text { Cepheids, } \gamma=-0.24 \\
{[\mathrm{Fe} / \mathrm{H}]=-1.6, \mathrm{E} . \mathrm{B}, \omega \text { Cen }} \\
{[\mathrm{M} / \mathrm{H}]=-1.2, \mathrm{E} . \mathrm{B}, \omega \text { Cen }} \\
\text { Six galaxies } \\
\text { RR Lyrae, LMC scale } \\
T \text { magnitude } \\
\text { F814W, model } \\
\text { NGC } 4258 \text { and the LMC }\end{array}$ \\
\hline
\end{tabular}

${ }^{\mathrm{a}} \mathrm{Col}=(\mathrm{F} 475 \mathrm{~W}-\mathrm{F} 814 \mathrm{~W})_{0}$.

$I$-band magnitude of the TRGB, $M_{I, T R G B}=-4.0 \pm 0.1$ mag, for metal poor stellar systems $([\mathrm{Fe} / \mathrm{H}]<-0.7)$. Salaris \& Cassisi (1997) derived a TRGB calibration having quadratic terms of metallicity from their theoretical stellar models. Absolute $I$-band magnitude of the TRGB at the fiducial metallicity, $[\mathrm{M} / \mathrm{H}]=-1.2$, is estimated to be $-4.16 \mathrm{mag}$, slightly brighter than that of Lee et al. (1993). This discrepancy is coming from the different estimation of the TRGB bolometric luminosity (Ferrarese et al. 2000). Ferrarese et al. (2000) calibrated the TRGB luminosity from the Cepheid distance estimations of nine galaxies in the Local Group. Absolute $I$-band magnitudes of the TRGB, -3.99 and $-4.06 \mathrm{mag}$ with an uncertainty of $\sim 0.15 \mathrm{mag}$, were derived depending on the adoption of metallicity dependence of Cepheid variables $\left(\gamma=\delta(m-M)_{0} / \delta[\mathrm{O} / \mathrm{H}]=0.00\right.$ and -0.24 , respectively).

Later Bellazzini et al. 2001, 2004) and Bellazzini (2008) presented another set of the TRGB calibration based on the larger sample of the Milky Way globular clusters: $M_{\text {bol,TRGB }}=-0.12[\mathrm{Fe} / \mathrm{H}]-3.76$ and $M_{\text {LTRGB }}=0.14[\mathrm{Fe} / \mathrm{H}]^{2}+0.48[\mathrm{Fe} / \mathrm{H}]-3.66$. Bellazzini et al. (2001) also presented another calibra- tion based on $\omega$ Centauri for which a direct distance estimate based on one eclipsing binary star (Thompson et al. 2001) is available. Adopting a metallicity of $[\mathrm{Fe} / \mathrm{H}]=$ -1.7 for $\omega$ Centauri, they derived $M_{\text {I,TRGB }}=-4.04 \pm$ 0.12 where the error is dominated by the distance measurement error for $\omega$ Centauri. This error has been used a value for the systematic error for the TRGB calibration (Mager et al. 2008; Conn et al. 2011; Shappee \& Stanek 2011; Lee \& Jang 2012, 2013; Jang \& Lee 2014, 2015; Lee \& Jang 2016). This value of the calibration is consistent with that from the quadratic calibration for $[\mathrm{Fe} / \mathrm{H}]$ $=-1.7$ in this study, $M_{\text {I.TRGB }}=-4.02$.

On the other hand Rizzi et al. (2007) provided a linear color dependence of the TRGB from the analysis of HST photometry data for six nearby galaxies: $M_{I, T R G B}=$ $-4.05+0.217\left[(V-I)_{0}-1.6\right]$. They constrained the zeropoint by using five Local Group galaxies, whose distances are obtained from the horizontal branch stars. Adopting the calibration of the horizontal branch stars given by Carretta et al. (2000), they derived a zero-point value, $-4.05 \pm 0.02 \mathrm{mag}$ at a fiducial color, $(V-I)_{0}=1.6$ (corresponding to $[\mathrm{Fe} / \mathrm{H}]=-1.5$ ). The systematic error of this value is mainly due to the uncertainty in the mean absolute magnitude of the horizontal branch stars, which 


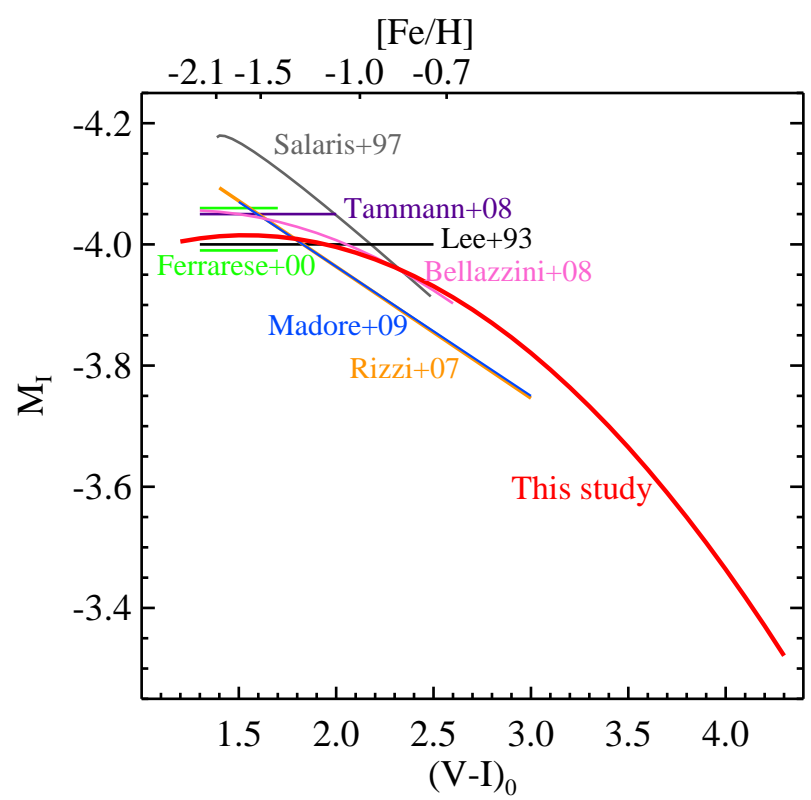

FIG. 13. - A comparison of the TRGB calibrations in $M_{I}-(V-$ $I)_{0}$ domain in this study and the previous studies (Lee et al. 1993. Salaris \& Cassisi 1997; Ferrarese et al. 2000; Rizzi et al. 2007; Bellazzini| 2008; Tammann et al.|2008; Madore et al.|2009).

is $\sim 0.1 \mathrm{mag}$ (Carretta et al. 2000).

Tammann et al. (2008) compiled the data for 24 galaxies that have estimates for both the TRGB magnitudes and the RR Lyrae distances in the literature, and derived an absolute magnitude of the TRGB, $M_{I, T R G B}=-4.05$ mag with a mean error of 0.02 mag. The systematic error of this value is mainly due to the uncertainty in the mean magnitude of the RR Lyrae, which is $\sim 0.1 \mathrm{mag}$ (Popowski \& Gould 1998a, b).

Madore et al. (2009) described a novel approach for a robust determination of the TRGB magnitude by introducing a new magnitude system, $T$ magnitude, which is a color (metallicity) corrected $I$-band magnitude: $T=$ $I_{0}-\beta\left((V-I)_{0}-1.5\right)$. They adopted a value for the slope, $\beta=0.20 \pm 0.05$ for $1.5<(V-I)_{0}<3.0$, from a linear approximation of the color-luminosity relation given by Bellazzini et al. (2001, 2004). They showed that the $T$ magnitude works well in the case of NGC 4258 (Mager et al. 2008; Madore et al. 2009).

Recently Fusco et al. (2012) carried out a TRGB calibration with the BaSTI stellar models (Pietrinferni et al. 2004, 2006), updating the conductive opacity evaluations provided by Cassisi et al. (2007). They presented variations of the TRGB magnitude as a function of metallicity and F475W - F814W color in their Figure 4. At the fiducial metallicity $([\mathrm{Fe} / \mathrm{H}]=-1.6)$, their calibration yields a value, $M_{\mathrm{F} 814 \mathrm{~W}, \mathrm{TRGB}}=-4.13$, which is slightly brighter than other calibrations.

In Figure 13, we compare our TRGB calibration with the previous ones in the $M_{I}-(V-I)_{0}$ domain. In the figure we set line lengths to be consistent with the color range used for each TRGB calibration. In the upper axis of the figure we also marked the metallicity values of the TRGB using the relation between $(V-I)_{0}$ and $[\mathrm{Fe} / \mathrm{H}]$ for $[\mathrm{Fe} / \mathrm{H}]<-0.7$, given by Bellazzini et al. (2001) $\left((V-I)_{0, T R G B}=0.581[\mathrm{Fe} / \mathrm{H}]^{2}+2.742[\mathrm{Fe} / \mathrm{H}]+\right.$ 4.013). Several important features are seen in this fig-

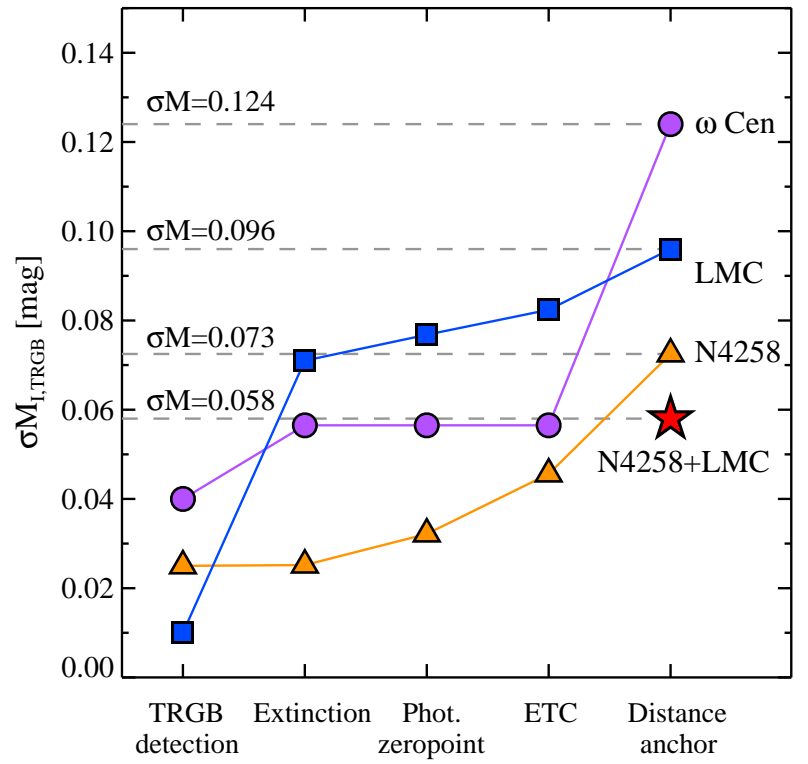

FIG. 14.- Propagation of uncertainties in the zero-point calibration of the TRGB. Note that the previous calibration based on $\omega$ Centauri gives a zero-point uncertainty of 0.124 mag (Bellazzini et al. 2001, 2004). When NGC 4258 and LMC are used as a distance anchor, uncertainties are estimated to be 0.073 and $0.096 \mathrm{mag}$, respectively. Combining these two anchors (NGC 4258 and LMC) provides the most accurate calibration with an uncertainty of $0.058 \mathrm{mag}$ (red starlet), $53 \%$ smaller than the value given by Bellazzini et al. (2001, 2004). Individual error values are listed in Table 4

ure. First, the revised TRGB calibration derived in this study (red line) covers a much wider color range, $1.2 \lesssim(V-I)_{0} \lesssim 4.3$, than other calibrations. Second, our TRGB calibration shows a color-magnitude relation that is relatively flat at the blue end and steep at the red color range. Some of the previous calibrations adopted either a flat relation (Lee et al. 1993; Ferrarese et al. 2000; Tammann et al. 2008) or color-dependent relations (Madore et al. 2009; Rizzi et al. 2007; Salaris \& Cassisi 1997). Third, the color dependence at the color range of $2.0 \lesssim(V-I)_{0} \lesssim 3.0$ derived in this study is similar to the results given by Salaris \& Cassisi (1997), Madore et al. (2009), Rizzi et al. (2007), and Bellazzini (2008). Fourth, our calibration shows an absolute magnitude of the TRGB, $M_{I}=-4.016 \pm 0.058$, at a fiducial color, $(V-I)_{0}=1.5$. It agrees well with the values from all of the previous calibrations within uncertainties.

Figure 14 shows the propagation of the zero-point uncertainties in the previous calibration based on $\omega$ Centauri (circles, Bellazzini et al. (2001, 2004)) and the revised $Q T$ calibrations based on NGC 4258 and the LMC in this study (triangles and squares). Four main sources of uncertainties (TRGB detection, extinction, photometric zero-point, and distance anchor uncertainties) and additional sources of uncertainties (ETC, which includes color transformation, photometric transformation, $Q T$ transformation uncertainties) listed in Table 4 are indicated. We calculated cumulative errors of each source (a quadratic sum of uncertainties including all the previous sources), according to this order. Bellazzini et al. (2001, 2004) presented a luminosity calibration of the TRGB based on the distance to $\omega$ Centauri, and derived a total uncertainty, 0.124 mag from the quadratic sum of 


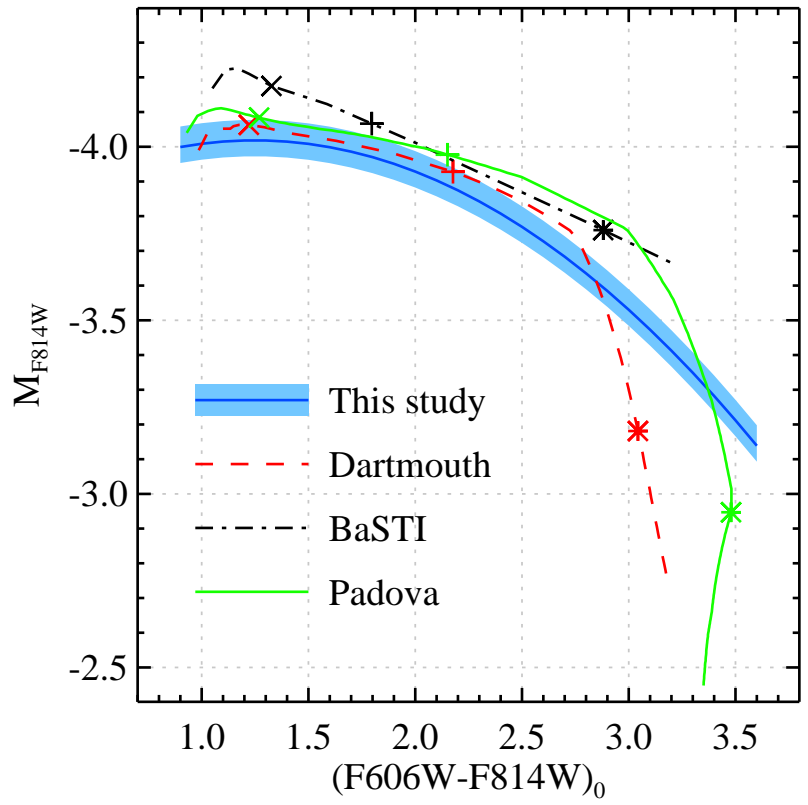

FIG. 15. - A comparison of the revised TRGB calibration (blue solid line with shaded region) with the TRGB from 12 Gyr stellar models: Dartmouth (Dotter et al. 2008) (red dashed line), BasTI (Pietrinferni et al. 2004, 2006) (black dot-dashed line), and Padova (Girardi et al. 2000; Marigo et al. 2013) (green solid line). Cross, plus, and asterisk symbols in each stellar model indicate $[\mathrm{M} / \mathrm{H}]=$ $-1.0,-0.5$, and 0.0 , respectively. The shaded region indicates a zero-point uncertainty, $0.046 \mathrm{mag}$, in this study.

three uncertainties: $0.04,0.04$, and $0.11 \mathrm{mag}$ for extinction, TRGB detection, and distance anchor uncertainties, respectively. They did not provide any uncertainty for the photometric zero-point. On the other hand, the revised TRGB calibration in this study yields total systematic uncertainty of $0.073 \mathrm{mag}$ from NGC 4258 and $0.096 \mathrm{mag}$ from the LMC as described in Section 3. A weighted mean of these two calibrations gives systematic uncertainty of $0.058 \mathrm{mag}$, which is about a half of the uncertainty given by Bellazzini et al. (2001, 2004). This value represents the systematic error of the revised TRGB calibration in this study.

\subsection{Comparison with Stellar Isochrones}

In Figure 15 we compare the empirical calibration of the TRGB (solid line with shaded region) derived in this study with the TRGB from three theoretical stellar models: Dartmouth (Dotter et al. 2008) (dashed line), BasTI (Pietrinferni et al. 2004, 2006) (dot-dashed line) , and Padova (Girardi et al. 2000; Marigo et al. 2013) (solid line). We set age of $12 \mathrm{Gyr}$ and metallicity range of $[\mathrm{M} / \mathrm{H}]=-2.2$ to +0.2 for stellar models. A fixed alpha element abundance, $[\alpha / F e]=+0.2$, was adopted for the Dartmouth model.

A few notable features are seen in this figure. First, the blue color range $\left((\mathrm{F} 606 \mathrm{~W}-\mathrm{F} 814 \mathrm{~W})_{0} \lesssim 1.6\right)$ of the empirical calibration shows a good agreement with those of the Dartmouth and Padova models. In the case of the BasTI model, however, it shows $0.20 \sim 0.15 \mathrm{mag}$ systematic offset and is steeper than those of other calibrations. Second, all four calibrations show a good agreement at $(\mathrm{F} 606 \mathrm{~W}-\mathrm{F} 814 \mathrm{~W})_{0} \sim 2.0$, which corresponds to $[\mathrm{M} / \mathrm{H}] \sim-0.5$. Third, a significant dispersion is seen at the red color range $\left((\mathrm{F} 606 \mathrm{~W}-\mathrm{F} 814 \mathrm{~W})_{0} \gtrsim 2.7\right)$.
The BasTI model gives relatively bright TRGB luminosity and a shallower color dependence. Dartmouth and Padova models show much steeper than others. Our empirical calibration of the TRGB will be very useful for improving the modelling of stellar evolution in the future.

\subsection{Implications on the Distance Scale and the Hubble Constant}

We obtained the zero-point of the TRGB, $M_{Q T, T R G B}=-4.023 \pm 0.073$ from NGC 4258 and $M_{Q T, T R G B}=-4.004 \pm 0.096$ from the LMC. The zero-point difference is $\Delta M_{Q T, T R G B}=-0.019 \pm 0.121$. Thus, the two zero-point values agree very well. From the TRGB magnitudes of NGC 4258 and the LMC, the distance modulus difference between two galaxies is obtained, $\Delta(m-M)_{0}=10.874 \pm 0.083$. This value shows excellent agreement with the value from the Cepheid variables, $\Delta(m-M)_{0}=10.88 \pm 0.04_{r} \pm 0.05_{s}$ (Macri et al. 2006). If we adopt the TRGB zero-point from the LMC, the TRGB distance to NGC 4258 is estimated to be $(m-M)_{0}=29.368 \pm 0.023_{r} \pm 0.096_{s}$, consistent with the recent megamaser distance to NGC 4258, $(m-M)_{0}=29.404 \pm 0.049_{r} \pm 0.043_{s}$ (Humphreys et al. 2013) and $(m-M)_{0}=29.387 \pm 0.049_{r} \pm 0.029_{s}$ (Riess et al. 2016).

The revised TRGB calibration derived in this study enables to determine more precise TRGB distances to nearby galaxies and a corresponding value of the Hubble constant. There have been several studies to determine the Hubble constant based on the TRGB (Ferrarese et al. 2000; Mould \& Sakai 2009a,b; Tammann et al. 2008; Hislop et al.|2011; Lee \& Jang 2012; Tammann \& Reindl 2013; Lee \& Jang 2013; Jang \& Lee 2015). Most of these studies were based on the zero-point of the TRGB, $M_{I}=-4.0 \sim-4.1 \mathrm{mag}$ with a systematic uncertainty of $0.10 \sim 0.12 \mathrm{mag}$. The revised TRGB calibration combined with both NGC 4258 and LMC anchors yields a TRGB zero-point of $M_{Q} T=-4.016 \pm 0.058 \mathrm{mag}$, slightly fainter and much more accurate than those of previous calibrations. Thus, the value of the Hubble constant derived in the previous studies can be slightly increased if the revised TRGB calibration is used.

Our previous studies, Lee \& Jang (2012, 2013) and Jang \& Lee (2015), presented a value of the Hubble constant, $H_{0}=69.8 \pm 2.6$ (random) \pm 3.9 (systematic) $\mathrm{km} \mathrm{s}^{-1}$ $\mathrm{Mpc}^{-1}$ and $H_{0}=72.2 \pm 3.3$ (random) \pm 4.0 (systematic) $\mathrm{km} \mathrm{s}^{-1} \mathrm{Mpc}^{-1}$ based on five and three TRGB calibrated SNe Ia. In these studies we used the old TRGB calibration given by Rizzi et al. (2007) and the systematic error of $0.12 \mathrm{mag}$ given by Bellazzini et al. (2001). If we apply the revised TRGB calibration including the $Q T$ magnitude and updated zero-point $\left(M_{Q T, T R G B}=\right.$ $-4.016 \pm 0.058 \mathrm{mag})$, then the value of $H_{0}$ would be increased by $\sim 1 \mathrm{~km} \mathrm{~s}^{-1} \mathrm{Mpc}^{-1}$ and the systematic uncertainty would be decreased by a factor of 2 . A detailed analysis of the TRGB distances to eight SN Ia host galaxies and the Hubble constant based on this new calibration will be presented in our upcoming paper (Jang\&Lee 2016, manuscript submitted).

\section{SUMMARY AND CONCLUSION}


The TRGB has been used as a reliable distance indicator for resolved stellar systems. However, its calibration needs to be improved to derive more precise distances to nearby galaxies and corresponding value of the Hubble constant. We present a revised TRGB calibration including the color dependence and zero-point calibrations, accurate to $0.058 \mathrm{mag}$ ( $2.7 \%$ of distance). Primary results are as follows.

- We obtained deep photometry of the resolved stars in eight nearby galaxies from the archival $H S T / A C S$ images. By applying quantitative TRGB detections on $\mathrm{F} 814 \mathrm{~W}_{0}-(\mathrm{F} 606 \mathrm{~W}-\mathrm{F} 814 \mathrm{~W})_{0}$ CMDs, we derived color-luminosity relation of the TRGB, which is described by a quadratic equation.

- From the photometry of two nearby globular clusters (NGC 2419 and 47 Tuc), we derived color transformation between $\mathrm{F} 555 \mathrm{~W}-\mathrm{F} 814 \mathrm{~W}$ and F606W - F814W in ACS/WFC system.

- The zero-point of the TRGB was determined from the photometry of two nearby galaxies, NGC 4258 and the LMC, to which geometric distances are known.

- We carried out PSF photometry using five different reduction methods on the HST/ACS field of NGC 4258 and compared the results. We found that output magnitudes agree well within 0.06 mag at the TRGB magnitude level. Especially, F814W band photometry shows excellent agreement within 0.02 mag, regardless of reduction methods.

- We provide the revised TRGB calibration in several filter systems including Johnson-Cousins, ACS/WFC, WFC3/UVIS, and WFPC2, as listed in Table 6 .

The authors thank the anonymous referee for the useful comments that improved the original manuscript. I.S.Jang thank Maria-Rosa L. Cioni and Jesper Storm for helpful discussions and comments. This work was supported by the National Research Foundation of Korea (NRF) grant funded by the Korea Government (MSIP) (No. 2012R1A4A1028713). This paper is based on image data obtained from the Multimission Archive at the Space Telescope Science Institute (MAST).

\section{REFERENCES}

Argon, A. L., Greenhill, L. J., Reid, M. J., Moran, J. M., \& Humphreys, E. M. L. 2007, ApJ, 659, 1040

Baade, W. 1944, ApJ, 100, 137

Beaton, R. L., Freedman, W. L., Madore, B. F., et al. 2016, arXiv: 1604.01788

Bellazzini, M., Ferraro, F. R., \& Pancino, E. 2001, ApJ, 556, 635

Bellazzini, M., Ferraro, F. R., Sollima, A., Pancino, E., \& Origlia L. 2004, A\&A, 424, 199

Bellazzini, M. 2008, Mem. Soc. Astron. Italiana, 79, 440

Bennett, C. L., Larson, D., Weiland, J. L., et al. 2013, ApJS, 208 20

Bennett, C. L., Larson, D., Weiland, J. L., \& Hinshaw, G. 2014 ApJ, 794, 135

Carretta, E., Gratton, R. G., Clementini, G., \& Fusi Pecci, F. 2000, ApJ, 533, 215

Cassisi, S., Potekhin, A. Y., Pietrinferni, A., Catelan, M., \& Salaris, M. 2007, ApJ, 661, 1094

Conn, A. R., Lewis, G. F., Ibata, R. A., et al. 2011, ApJ, 740, 69

Conn, A. R., Ibata, R. A., Lewis, G. F., et al. 2012, ApJ, 758, 11

Da Costa, G. S., \& Armandroff, T. E. 1990, AJ, 100, 162

Dalcanton, J. J., Williams, B. F., Seth, A. C., et al. 2009, ApJS, 183,67

de Grijs, R., Wicker, J. E., \& Bono, G. 2014, AJ, 147, 122

Dolphin, A. E. 2000, PASP, 112, 1383

Dotter, A., Chaboyer, B., Jevremović, D., et al. 2008, ApJS, 178, 89

Ferrarese, L., Mould, J. R., Kennicutt, R. C., Jr., et al. 2000, ApJ, 529, 745

Freedman, W. L. 1988, ApJ, 326, 691

Freedman, W. L. 1988, AJ, 96, 1248

Freedman, W. L. 1989, AJ, 98, 1285

Freedman, W. L., Madore, B. F., Gibson, B. K., et al. 2001, ApJ, 553,47

Freedman, W. L., \& Madore, B. F. 2010, ARA\&A, 48, 673

Freedman, W. L., Madore, B. F., Scowcroft, V., et al. 2012, ApJ, 758,24

Frogel, J. A., Cohen, J. G., \& Persson, S. E. 1983, ApJ, 275, 773

Fusco, F., Buonanno, R., Bono, G., et al. 2012, A\&A, 548, A129

Gerke, J. R., Kochanek, C. S., Prieto, J. L., Stanek, K. Z., \& Macri, L. M. 2011, ApJ, 743, 176

Girardi, L., Bressan, A., Bertelli, G., \& Chiosi, C. 2000, A\&AS, 141,371

Górski, M., Pietrzyński, G., Gieren, W.. et al. 2016, AJ, 151, 167

Green, E. M., Demarque, P., \& King, C. R. 1987, The revised Yale isochrones and luminosity functions, New Haven: Yale Observatory, 1987,

Greenhill, L. J., Jiang, D. R., Moran, J. M., et al. 1995, ApJ, 440, 619

Haschke, R., Grebel, E. K., \& Duffau, S. 2011, AJ, 141, 158
Herrnstein, J. R., Moran, J. M., Greenhill, L. J., et al. 1999, Nature, 400, 539

Hislop, L., Mould, J., Schmidt, B., et al. 2011, ApJ, 733, 75

Holtzman, J. A., Gallagher, J. S., III, Cole, A. A., et al. 1999, AJ, 118,2262

Humphreys, E. M. L., Argon, A. L., Greenhill, L. J., Moran, J. M., \& Reid, M. J. 2005, Future Directions in High Resolution Astronomy, 340, 466

Humphreys, E. M. L., Reid, M. J., Greenhill, L. J., Moran, J. M., \& Argon, A. L. 2008, ApJ, 672, 800

Humphreys, E. M. L., Reid, M. J., Moran, J. M., Greenhill, L. J., \& Argon, A. L. 2013, ApJ, 775, 13

Jang, I. S., \& Lee, M. G. 2014, ApJ, 792, 52

Jang, I. S., \& Lee, M. G. 2015, ApJ, 807, 133 (Paper III)

Lee, M. G., Freedman, W. L., \& Madore, B. F. 1993, ApJ, 417, 553

Lee, M. G., \& Jang, I. S. 2012, ApJ, 760, L14 (Paper I)

Lee, M. G., \& Jang, I. S. 2013, ApJ, 773, 13 (Paper II)

Lee, M. G., \& Jang, I. S. 2016, ApJ, 822, 70

Macri, L. M., Stanek, K. Z., Bersier, D., Greenhill, L. J., \& Reid, M. J. 2006, ApJ, 652, 1133

Macri, L. M., Ngeow, C.-C., Kanbur, S. M., Mahzooni, S., \& Smitka, M. T. 2015, AJ, 149, 117

Madore, B. F., Mager, V., \& Freedman, W. L. 2009, ApJ, 690, 389

Mager, V. A., Madore, B. F., \& Freedman, W. L. 2008, ApJ, 689 , 721

Makarov, D., Makarova, L., Rizzi, L., et al. 2006, AJ, 132, 2729

Marigo, P., Bressan, A., Nanni, A., Girardi, L., \& Pumo, M. L. 2013, MNRAS, 434, 488

Méndez, B., Davis, M., Moustakas, J., et al. 2002, AJ, 124, 213

Miyoshi, M., Moran, J., Herrnstein, J., et al. 1995, Nature, 373, 127

Mould, J. R., Kristian, J., \& Da Costa, G. S. 1983, ApJ, 270, 471

Mould, J., Kristian, J., \& Da Costa, G. S. 1984, ApJ, 278, 575

Mould, J., \& Kristian, J. 1986, ApJ, 305, 591

Mould, J., \& Sakai, S. 2009, ApJ, 694, 1331

Mould, J., \& Sakai, S. 2009, ApJ, 697, 996

Pietrinferni, A., Cassisi, S., Salaris, M., \& Castelli, F. 2004, ApJ, 612,168

Pietrinferni, A., Cassisi, S., Salaris, M., \& Castelli, F. 2006, ApJ, 642,797

Pietrzyński, G., Graczyk, D., Gieren, W., et al. 2013, Nature, 495, 76

Planck Collaboration, Ade, P. A. R., Aghanim, N., et al. 2014, A\&A, 571, AA16

Planck Collaboration, Ade, P. A. R., Aghanim, N., et al. 2015, arXiv:1502.01589

Popowski, P., \& Gould, A. 1998, ApJ, 506, 259

Popowski, P., \& Gould, A. 1998, ApJ, 506, 271 
Radburn-Smith, D. J., de Jong, R. S., Seth, A. C., et al. 2011, ApJS, 195, 18

Riess, A. G., Macri, L., Casertano, S., et al. 2011, ApJ, 730, 119

Riess, A. G., Macri, L., Casertano, S., et al. 2012, ApJ, 752, 76

Riess, A. G., Macri, L. M., Hoffmann, S. L., et al. 2016, arXiv: 1604.01424

Rizzi, L., Tully, R. B., Makarov, D., et al. 2007, ApJ, 661, 815

Saha, A., Shaw, R. A., Claver, J. A., \& Dolphin, A. E. 2011, PASP, 123, 481

Sakai, S., Madore, B. F., \& Freedman, W. L. 1996, ApJ, 461, 713

Salaris, M., \& Cassisi, S. 1997, MNRAS, 289, 406

Salaris, M., \& Girardi, L. 2005, MNRAS, 357, 669

Sandage, A. R. 1971, Study Week on Nuclei of Galaxies, 601

Sandage, A., Tammann, G. A., Saha, A., et al. 2006, ApJ, 653, 843
Savitzky, A., \& Golay, M. J. E. 1964, Analytical Chemistry, 36, 1627

Schaefer, B. E. 2008, AJ, 135, 112

Schlafly, E. F., \& Finkbeiner, D. P. 2011, ApJ, 737, 103

Shappee, B. J., \& Stanek, K. Z. 2011, ApJ, 733, 124

Sirianni, M., Jee, M. J., Benítez, N., et al. 2005, PASP, 117, 1049

Stetson, P. B. 1987, PASP, 99, 191

Tammann, G. A., Sandage, A., \& Reindl, B. 2008, ApJ, 679, 52

Tammann, G. A., \& Reindl, B. 2013, A\&A, 549, A136

Thompson, I. B., Kaluzny, J., Pych, W., et al. 2001, AJ, 121, 3089

Tully, R. B., Courtois, H. M., Dolphin, A. E., et al. 2013, AJ, 146, 86

Ulaczyk, K., Szymański, M. K., Udalski, A., et al. 2012, Acta Astron, 62, 247

Yang, S.-C., Wagner-Kaiser, R., Sarajedini, A., Kim, S. C., \& Kyeong, J. 2014, ApJ, 784, 76 